\title{
The long-time chronoamperometric current at an inlaid disk electrode
}

\author{
Christopher G. Bell ${ }^{\mathrm{a}, 1, *}$, Peter D. Howell ${ }^{\mathrm{b}}$, Howard A. Stone ${ }^{\mathrm{c}}$, Wen-Jei Lim ${ }^{\mathrm{a}}$, Jennifer \\ H. Siggers ${ }^{\mathrm{a}}$ \\ ${ }^{a}$ Department of Bioengineering, Imperial College London, South Kensington Campus, London, SW7 2AZ, \\ $U K$ \\ ${ }^{b}$ Mathematical Institute, University of Oxford, 24-29 St Giles', Oxford, OX1 3LB, UK \\ ${ }^{c}$ Department of Mechanical and Aerospace Engineering, Princeton University, Princeton, New Jersey \\ 08544, United States
}

\begin{abstract}
Existing analytical solutions for the long-time chronoamperometric current response at an inlaid disk electrode are restricted to diffusion-limited currents due to extreme polarisation or reversible kinetics at the electrode surface. In this article, we derive an approximate analytical solution for the long-time-dependent current when the kinetics of the redox reaction at the electrode surface are quasi-reversible and the diffusion coefficients of the oxidant and reductant are different. We also detail a novel method for calculating the steady-state current. We show that our new method encapsulates and extends the existing solutions, and agrees with numerically simulated currents.
\end{abstract}

Keywords: chronoamperometry, disk, ultramicroelectrode, quasi-reversible, analytical

\section{Introduction}

Microdisk electrodes, and in particular ultra-microdisk electrodes, are popularly used for electrochemical investigations, since they possess many advantages [1-3]. A microdisk electrode is a conducting disk embedded in an insulating plane, and is easily fabricated by slicing through an insulated wire. Due to the geometry of the electrode, mass transport is enhanced at the edge of the disk, and the current scales with the radius of the disk rather than the area. The effects of ohmic drop and double-layer capacitance are reduced, and the behaviour of electrochemical systems can be investigated over very small time- and length-scales. Miniaturisation of the electrode allows accurate information to be obtained about reactions with fast kinetics, which would be impossible to distinguish at larger electrodes, [4]. Since this type of electrode is

\footnotetext{
${ }^{*}$ Corresponding author

Email address: bell @maths . ox . ac.uk (Christopher G. Bell)

${ }^{1}$ Present address: Mathematical Institute, University of Oxford, 24-29 St Giles', Oxford, OX1 3LB, UK, Tel: +44 (0)1865 273525, Fax: +44 (0)1865 273583
} 
so widely used, theoretical research is vital to understand how the current response should behave. Theoretical investigations are complicated by the different boundary conditions on the electrode and the insulator, which results in a discontinuity in the flux normal to the surface at the electrode edge.

The general problem involves two redox species, Ox and Red, diffusing above a disk electrode with radius $\tilde{a}$ inlaid in an insulating plane. The diffusion coefficients for Ox and Red are denoted $\tilde{D}_{O}$ and $\tilde{D}_{R}$ respectively, and they are not generally equal. Provided the potential at the electrode is stepped to a constant value, the following redox reaction occurs at the electrode and produces a chronoamperometric current:

$$
\mathrm{Ox}+n \mathrm{e} \underset{\tilde{k}_{b}}{\stackrel{\tilde{k}_{f}}{\rightleftharpoons}} \operatorname{Red}
$$

where the forward and backward reaction rates, $\tilde{k}_{f}$ and $\tilde{k}_{b}$, are constant. If the effects of migration and natural convection can be neglected, then the current produced is a function of the rate of mass transport to the electrode due to diffusion and the rate of the reaction itself. Eventually the current reaches a steady state.

Analytically, investigations into the current produced at a disk electrode started with the steady-state problem. The earliest recorded solutions in the electrochemical literature date back to Newman [5] and Saito [6], who reported the formula for the diffusionlimited current due to extreme polarization, which was also well-known from potential theory [7]. For reversible kinetics, where the Nernst equation applies at the electrode surface, the analytical formula for the resulting diffusion-limited current is also wellknown, cf. Bond et al. [4] (using the properties of discontinuous integrals of Bessel functions) and Oldham [8] (using spheroidal coordinates). More generally, reversible diffusion-limited currents occur whenever the following dimensionless parameter is infinite (cf. Phillips [9]):

$$
\beta=\frac{\tilde{k}_{f} \tilde{a}}{\tilde{D}_{O}}+\frac{\tilde{k}_{b} \tilde{a}}{\tilde{D}_{R}}
$$

If $\beta$ is finite, then the reaction at the electrode is quasi-reversible. In this case, the steady-state current depends on a function of $\beta$, which generally must be calculated numerically. Analytical approximations have been derived by Phillips [9] for large $\beta$ (when the current is close to diffusion-limited), and by Bender and Stone [10] for small $\beta$. Bender and Stone [10] also used a Green's function approach to derive an integral equation for the current for any $\beta$, which they solved numerically. Aoki et al. [11] used the Wiener-Hopf method to show that the steady-state current for a quasireversible reaction can be calculated by solving a truncated infinite set of simultaneous equations. Three other approaches have been illustrated in the literature, namely that of Bond et al. [4] (using the properties of discontinuous integrals of Bessel functions), Fleischmann, Daschbach and Pons $[12,13]$ (using the Neumann integral theorem) and Baker and Verbrugge $[14,15]$ (using an integral equation written in terms of elliptic integrals, similar to the approach of Bender and Stone [10]). Oldham and Zoski [16] demonstrated that these three approaches are fundamentally similar and showed that they yield the same numerical values.

The behaviour of the transient current before the system reaches steady-state, corresponding to a chronoamperometric experiment, is also of interest to researchers. For 
reversible reactions, and assuming that the diffusion coefficients of the oxidant and reductant are equal, Aoki and Osteryoung [17, 18] used the Wiener-Hopf procedure to develop approximate series expansions for the transient currents at short time and long time; the long-time series was subsequently corrected by Shoup and Szabo [19]. As part of a more general article on the long-time transient currents to microelectrodes of arbitrary shape, Phillips [20] showed that, in the special case of an inlaid disk, his solution agreed with Shoup and Szabo's correction. Due to an approximation in Aoki and Osteryoung's analysis [17], there was some doubt about the third term in the short-time series [18, 19], and Phillips and Jansons [21] derived a corrected version of the series. Oldham [22] found the first two terms in the short-time series for the diffusion-limited current in the case of extreme polarisation. Rajendran and Sangaranarayanan [23] also derived five- and four-term series respectively for the diffusionlimited currents at short- and long-time using results from scattering analogue theory, valid for equal diffusion coefficients. Fleischmann and coworkers also considered the chronoamperometric response of a disk electrode at extreme polarisation. In [24], they find an approximate solution in the Laplace-transformed variable, which satisfies the constant concentration boundary condition on average across the disk; and, in [25], they use Neumann's integral theorem to find a series solution (which they also extend to irreversible reactions), the time-dependent coefficients of which must be determined from a system of complicated equations.

A number of different numerical approaches have been developed to investigate both the steady-state current and the transient chronoamperometric current. Gavaghan $[26,27]$ developed a finite-difference approach using a spatial grid expanding exponentially from the electrode edge. Harriman, Gavaghan, Süli et al. [28-30] used an adaptive finite-element approach. Amatore, Oleinick and Svir [31-34] have described how to use quasi-conformal mapping techniques. Mirkin and Bard [35] showed how the transient current can be calculated from a multi-dimensional integral equation. Although extremely useful, these numerical simulations cannot provide the same direct insight as analytical solutions into how the current response depends on the underlying system parameters.

All of the analytical work on transient chronoamperometric currents descibed above only covers diffusion-limited currents, due to extreme polarisation or reversible kinetics, when the parameter $\beta$ is infinite. For the reversible kinetics/infinite- $\beta$ case, existing analysis also requires that the diffusion coefficients of the oxidant and the reductant are equal. In this article, we derive a two-term asymptotic series for the general long-time chronoamperometric current. For the reader who wishes to skip the detailed derivation, the final expression is given in equation (52). The solution extends the prior work described above to allow for quasi-reversible kinetics at the electrode and unequal diffusion coefficients. By 'long-time', we mean that the solution is valid for times, $\tilde{t}$, such that the following condition is satisfied:

$$
\tilde{t} \gg \max \left(\frac{\tilde{a}^{2}}{\tilde{D}_{O}}, \frac{\tilde{a}^{2}}{\tilde{D}_{R}}\right)
$$

We demonstrate that the solution encapsulates the existing solutions for the diffusionlimited currents, and we show that it agrees with numerically simulated values using 
Gavaghan's finite-difference method [26, 27]. The first term in the series is the steadystate current, and the second term is proportional to $\tilde{t}^{-1 / 2}$, and depends on the square of the steady-state current. As detailed above, solutions for the steady-state current for a quasi-reversible reaction are already known. However, whilst carrying out this research, we found a new solution for the steady-state current using Tranter's method [36], which exploits the properties of discontinuous integrals of Bessel functions, and we report this in Appendix A. The approach is similar to Bond et al. [4], but uses different weighting functions. The resulting truncated infinite system of equations to be solved is easy to implement, since the coefficients in the matrix are simple, and converge quickly.

\section{Theory}

\subsection{Problem statement and non-dimensionalisation}

A schematic of the dimensional theoretical problem is displayed in Figure 1 (tildes indicate dimensional variables). We consider a simple redox reaction (1) between two species, Ox and Red, diffusing in the half-space $\tilde{z}>0$, which exchange $n$ electrons at disk electrode placed in the plane $\tilde{z}=0$. The forward and backward rate constants of the reaction are denoted by $\tilde{k}_{f}$ and $\tilde{k}_{b}$ respectively, and we will assume that the electrode is held at a constant potential so that they are both constant. The inlaid disk electrode has its centre situated at $\tilde{r}=0, \tilde{z}=0$ and has radius $\tilde{r}=\tilde{a}(\mathrm{~m})$. If any effects due to migration and convection are neglected, then the concentrations of Ox and Red, $\tilde{C}_{O}(\tilde{r}, \tilde{z}, \tilde{t})$ and $\tilde{C}_{R}(\tilde{r}, \tilde{z}, \tilde{t})$, each satisfy the diffusion equation for $\tilde{z}>0$ with constant diffusion coefficients $\tilde{D}_{O}$ and $\tilde{D}_{R}\left(\mathrm{~m}^{2} \mathrm{~s}^{-1}\right)$, that is:

$$
\tilde{D}_{O} \nabla^{2} \tilde{C}_{O}=\frac{\partial \tilde{C}_{O}}{\partial \tilde{t}}, \quad \tilde{D}_{R} \nabla^{2} \tilde{C}_{R}=\frac{\partial \tilde{C}_{R}}{\partial \tilde{t}} .
$$

Initially the bulk concentration of each species is constant everywhere:

$$
\tilde{C}_{O}(\tilde{r}, \tilde{z}, 0)=\tilde{C}_{O}^{*}, \quad \tilde{C}_{R}(\tilde{r}, \tilde{z}, 0)=\tilde{C}_{R}^{*}
$$

We assume that the bulk concentrations remain undisturbed as the reaction at the electrode progresses, which provides the far-field boundary conditions

$$
\tilde{C}_{O} \rightarrow \tilde{C}_{O}^{*}, \quad \tilde{C}_{R} \rightarrow \tilde{C}_{R}^{*}, \quad \text { as } \tilde{r}^{2}+\tilde{z}^{2} \rightarrow \infty .
$$

On the electrode surface, the boundary conditions are given by the reaction at the surface and conservation of matter:

$$
\left.\begin{array}{l}
\tilde{D}_{O} \frac{\partial \tilde{C}_{O}}{\partial \tilde{z}}=\tilde{k}_{f} \tilde{C}_{O}-\tilde{k}_{b} \tilde{C}_{R}, \\
\tilde{D}_{O} \frac{\partial \tilde{C}_{O}}{\partial \tilde{z}}=-\tilde{D}_{R} \frac{\partial \tilde{C}_{R}}{\partial \tilde{z}},
\end{array}\right\} \quad \text { for } \tilde{r} \leq \tilde{a}, \tilde{z}=0 .
$$

There is no flux through the remainder of the surface, so that

$$
\tilde{D}_{O} \frac{\partial \tilde{C}_{O}}{\partial \tilde{z}}=\tilde{D}_{R} \frac{\partial \tilde{C}_{R}}{\partial \tilde{z}}=0, \quad \text { for } \tilde{r}>\tilde{a}, \tilde{z}=0
$$


The Faradaic current through the electrode is given by

$$
\tilde{I}(\tilde{t})=-2 \pi n F \tilde{D}_{O} \int_{0}^{\tilde{a}} \frac{\partial \tilde{C}_{O}}{\partial \tilde{z}}(\tilde{r}, 0, \tilde{t}) \tilde{r} \mathrm{~d} \tilde{r}
$$

where $F$ is Faraday's constant, and we recall that $n$ is the number of electrons transferred in the redox reaction.

To non-dimensionalise the problem, we choose the following scalings:

$$
\begin{gathered}
\tilde{r}=\tilde{a} r, \quad \tilde{z}=\tilde{a} z, \quad \tilde{t}=\frac{\tilde{a}^{2}}{\tilde{D}_{O}} t, \quad \tilde{k}_{f}=\frac{\tilde{D}_{O}}{\tilde{a}} k_{f}, \quad \tilde{k}_{b}=\frac{\tilde{D}_{O}}{\tilde{a}} k_{b} \\
\tilde{C}_{O}=\tilde{C}_{O}^{*}-\left(\frac{k_{f} \tilde{C}_{O}^{*}-k_{b} \tilde{C}_{R}^{*}}{k_{f}+k_{b} \mathcal{D}^{-1}}\right) C_{O}, \quad \tilde{C}_{R}=\tilde{C}_{R}^{*}-\left(\frac{k_{f} \tilde{C}_{O}^{*}-k_{b} \tilde{C}_{R}^{*}}{k_{f}+k_{b} \mathcal{D}^{-1}}\right) C_{R} \\
\tilde{I}=n F \tilde{a} \tilde{D}_{O}\left(\frac{k_{f} \tilde{C}_{O}^{*}-k_{b} \tilde{C}_{R}^{*}}{k_{f}+k_{b} \mathcal{D}^{-1}}\right) I
\end{gathered}
$$

where

$$
\mathcal{D}=\frac{\tilde{D}_{R}}{\tilde{D}_{O}}
$$

Then, in terms of the non-dimensional variables, the problem becomes:

$$
\nabla^{2} C_{O}=\frac{\partial C_{O}}{\partial t}, \quad \mathcal{D} \nabla^{2} C_{R}=\frac{\partial C_{R}}{\partial t}, \quad \text { in } z>0 .
$$

The initial conditions become:

$$
C_{O}(r, z, 0)=0, \quad C_{R}(r, z, 0)=0,
$$

and the far-field boundary conditions are

$$
C_{O} \rightarrow 0, \quad C_{R} \rightarrow 0, \quad \text { as } r^{2}+z^{2} \rightarrow \infty .
$$

On the electrode surface, the boundary conditions are:

$$
\left.\begin{array}{rl}
\frac{\partial C_{O}}{\partial z} & =\left(k_{f}+k_{b} \mathcal{D}^{-1}\right)\left(\frac{k_{f} C_{O}-k_{b} C_{R}}{k_{f}+k_{b} \mathcal{D}^{-1}}-1\right), \\
\frac{\partial C_{O}}{\partial z} & =-\mathcal{D} \frac{\partial C_{R}}{\partial z},
\end{array}\right\} \quad \text { for } r \leq 1, z=0 .
$$

On the remaining surface, the no-flux condition is:

$$
\frac{\partial C_{O}}{\partial z}=\frac{\partial C_{R}}{\partial z}=0, \text { for } r>1, z=0 .
$$

The dimensionless Faradaic current through the electrode is given by

$$
I(t)=2 \pi \int_{0}^{1} \frac{\partial C_{O}}{\partial z}(r, 0, t) r \mathrm{~d} r .
$$




\subsection{Steady-state problem}

The long-time solution of the time-dependent problem is highly reliant on the solution to the steady-state problem, which we consider here first. The steady-state concentrations, which we denote $C_{O}^{s s}$ and $C_{R}^{s s}$, satisfy Laplace's equation:

$$
\nabla^{2} C_{O}^{s s}=0, \quad \nabla^{2} C_{R}^{s s}=0,
$$

in $z>0$, along with the boundary conditions (14)-(16). It is simple to see that $C_{O}^{s s}+$ $\mathcal{D} C_{R}^{s s}=0$ for all $r, z$, so that solution of the steady-state problem reduces to solving the following problem for $C_{O}^{s s}$ :

$$
\begin{aligned}
& \nabla^{2} C_{O}^{s s}=0 \\
& z>0, \\
& C_{O}^{s s} \rightarrow 0 \\
& z \rightarrow \infty, \\
& \frac{\partial C_{O}^{s s}}{\partial z}=q(\beta ; r)=\left\{\begin{array}{ll}
0 & r>1, \\
\beta\left(C_{O}^{s s}-1\right) & r \leq 1,
\end{array} \quad z=0,\right.
\end{aligned}
$$

where the mass transfer coefficient $\beta$ is given by

$$
\beta=k_{f}+k_{b} \mathcal{D}^{-1} \text {. }
$$

(This is expression (2) written in non-dimensional variables.) The solution to this problem, $C_{O}^{s s}=C_{O}^{s s}(\beta ; r, z)$, depends parametrically on the single parameter $\beta$, and the steady-state current, $I_{s s}(\beta)$, is given by (cf. (17))

$$
I_{s s}(\beta)=\left.2 \pi \int_{0}^{1} \frac{\partial C_{O}^{s s}}{\partial z}\right|_{z=0} r \mathrm{~d} r=2 \pi \int_{0}^{1} q(\beta ; r) r \mathrm{~d} r .
$$

The steady-state current as a function of $\beta$ can be computed in a number of ways as described in the Introduction [4, 10-16]. We have found a new solution using Tranter's method [36], which we detail in Appendix A. This methodology uses the properties of discontinuous integrals of Bessel functions, and is similar to that employed by Bond et al. [4], but uses different weighting functions. Our methodology results in a simpler matrix equation to solve for the current and it converges very quickly as the size of the matrix is increased. The result is shown as a log-log plot in Figure 2, along with the small- and large- $\beta$ asymptotes. The small- $\beta$ asymptote was derived by Bender and Stone [10], and is given by:

$$
I_{s s}(\beta) \sim-\pi \beta+\frac{8}{3} \beta^{2}-2.294 \beta^{3}+1.969 \beta^{4}+O\left(\beta^{5}\right), \quad \text { as } \beta \rightarrow 0,
$$

while the large- $\beta$ asymptote was derived by Phillips [9] to be:

$$
I_{s s}(\beta)=-4\left(1-(\pi \beta)^{-1} \log \beta+o\left(\beta^{-1} \log \beta\right)\right), \quad \text { as } \beta \rightarrow \infty .
$$

By comparing their numerical solution to this asymptotic approximation, Bender and Stone [10] suggested that (23) can be improved by adding a numerically based correction of $O\left(\beta^{-1}\right)$, so that the asymptote is given by:

$$
I_{s s}(\beta)=-4\left(1-\beta^{-1}\left(\pi^{-1} \log \beta+0.725\right)+o\left(\beta^{-1} \log \beta\right)\right), \quad \text { as } \beta \rightarrow \infty .
$$


Nisanciog̈lu and Newman [37] found the coefficient of the extra $O\left(\beta^{-1}\right)$ term to be 0.708 .

Using a Green's function, the solution for $C_{O}^{s s}$ can be expressed in terms of the flux, $q(\beta ; r)$, defined in $(19 \mathrm{c})$, as [14, 38]:

$$
C_{O}^{s s}(\beta ; r, z)=-\frac{2}{\pi} \int_{0}^{1} \mathrm{~K}\left(\frac{4 r s}{(r+s)^{2}+z^{2}}\right) \frac{q(\beta ; s) s \mathrm{~d} s}{\sqrt{(r+s)^{2}+z^{2}}},
$$

where $\mathrm{K}(m)$ denotes a complete elliptic integral of the first kind ([39], p. 590, 17.3.1):

$$
\mathrm{K}(m)=\int_{0}^{\pi / 2} \frac{1}{\sqrt{1-m \sin ^{2} \theta}} \mathrm{d} \theta .
$$

Solution of the transient problem requires knowledge of the far-field behaviour of $C_{O}^{s s}(\beta ; r, z)$, which can be derived from (25) to be:

$$
\begin{array}{r}
C_{O}^{s s}(\beta ; r, z) \sim-\frac{I_{s s}(\beta)}{2 \pi \sqrt{r^{2}+z^{2}}}-\frac{\left(r^{2}-2 z^{2}\right)}{4\left(r^{2}+z^{2}\right)^{5 / 2}} \int_{0}^{1} q(\beta ; s) s^{3} \mathrm{~d} s+\cdots, \\
\text { as } r^{2}+z^{2} \rightarrow \infty .
\end{array}
$$

To leading order, the far-field influence of the disk is characterised entirely by the steady-state current $I_{s s}(\beta)$ and is equivalent to a point source of strength $I_{s s}(\beta)$.

Since $I_{s s}(\beta)$ is critical for the understanding of the current response, we have supplied a working curve for $I_{s s}(\beta)$ as a function of $\beta$ over the range $0 \leq \beta \leq 500$ in the Supplementary Information; details of the calculation are set out in Appendix A. For $\beta>500$, the asymptotic approximations (23) or (24) for large $\beta$ can be used.

\subsection{Asymptotic solution for the long-time transient behaviour}

To find the long-time solution, we perform a coordinate expansion for large $t$ by letting

$$
t=\frac{T}{\epsilon^{2}}
$$

where $T=O(1)$ and $\epsilon \ll 1$, so that the governing equations (12) become:

$$
\nabla^{2} C_{O}=\epsilon^{2} \frac{\partial C_{O}}{\partial T}, \quad \mathcal{D} \nabla^{2} C_{R}=\epsilon^{2} \frac{\partial C_{R}}{\partial T} .
$$

In dimensional terms, the condition $\epsilon \ll 1$ is equivalent to assuming condition (3) mentioned in the Introduction, and which we repeat here:

$$
\tilde{t} \gg \max \left(\frac{\tilde{a}^{2}}{\tilde{D}_{O}}, \frac{\tilde{a}^{2}}{\tilde{D}_{R}}\right) .
$$

For example, for a microdisk electrode with radius $\tilde{a} \approx 10^{-5} \mathrm{~m}$ and diffusion coefficients $\tilde{D}_{O}, \tilde{D}_{R} \approx 10^{-10} \mathrm{~m}^{2} \mathrm{~s}^{-1}$, this condition implies that the solution that we derive will be valid for time-scales $\tilde{t} \gg 1$ s. 
Since $\epsilon \ll 1$, there is an inner region near the disk where the concentrations are at a steady state to leading order, and a outer region far from the disk where the concentrations are time-dependent to leading order. We find solutions in both regions using approximate matched asymptotic expansions. This approach is similar to that used for the diffusion-limited current by Phillips [20], who performed the analysis in the Laplace-transform domain. In the inner region, the coordinate system is simply $(r, z)$ as defined above, and we denote the inner dependent variables by using the subscript $i$, so that they are $C_{O, i}(r, z, T)$ and $C_{R, i}(r, z, T)$. In the outer region, we define the coordinate system to be $(\hat{r}, \hat{z})$, where $\hat{r}=\epsilon r$ and $\hat{z}=\epsilon z$, and we denote the dependent variables using the subscript $o: C_{O, o}(\hat{r}, \hat{z}, T)$ and $C_{R, o}(\hat{r}, \hat{z}, T)$.

We expand the inner variables in the following perturbation series:

$$
\begin{aligned}
& C_{O, i}(r, z, T)=C_{O, i}^{(0)}(r, z)+\epsilon C_{O, i}^{(1)}(r, z, T)+\epsilon^{2} C_{O, i}^{(2)}(r, z, T)+\ldots, \\
& C_{R, i}(r, z, T)=C_{R, i}^{(0)}(r, z)+\epsilon C_{R, i}^{(1)}(r, z, T)+\epsilon^{2} C_{R, i}^{(2)}(r, z, T)+\ldots
\end{aligned}
$$

and the outer variables as:

$$
\begin{aligned}
& C_{O, o}(\hat{r}, \hat{z}, T)=\epsilon C_{O, o}^{(0)}(\hat{r}, \hat{z}, T)+\epsilon^{2} C_{O, o}^{(1)}(\hat{r}, \hat{z}, T)+\epsilon^{3} C_{O, o}^{(2)}(\hat{r}, \hat{z}, T)+\ldots \\
& C_{R, o}(\hat{r}, \hat{z}, T)=\epsilon C_{R, o}^{(0)}(\hat{r}, \hat{z}, T)+\epsilon^{2} C_{R, o}^{(1)}(\hat{r}, \hat{z}, T)+\epsilon^{3} C_{R, o}^{(2)}(\hat{r}, \hat{z}, T)+\ldots
\end{aligned}
$$

\subsubsection{Leading-order inner solution}

To leading order, the concentrations are at steady state in the vicinity of the electrode, so that $C_{O, i}^{(0)}(r, z)$ and $C_{R, i}^{(0)}(r, z)$ satisfy the steady-state problem discussed in Section 2.2. Hence

$$
C_{O, i}^{(0)}(r, z)=C_{O}^{s s}(\beta ; r, z)
$$

where the steady-state solution, $C_{O}^{s s}$, is given by expression (25), and the corresponding leading-order solution for $C_{R, i}^{(0)}$ is:

$$
C_{R, i}^{(0)}(r, z)=-\frac{1}{\mathcal{D}} C_{O}^{s s}(\beta ; r, z) .
$$

\subsubsection{Leading-order outer solution}

Now we apply Van Dyke's matching rule [40].Writing the inner solutions $C_{O, i}^{(0)}$ and $C_{R, i}^{(0)}$ in terms of the outer variables $\hat{r}$ and $\hat{z}$, and letting $\epsilon$ tend to zero, we find from (27) that

$$
\begin{aligned}
C_{O, i}^{(0)}(\hat{r}, \hat{z}) & =-\epsilon \frac{I_{s s}(\beta)}{2 \pi \hat{\rho}}+O\left(\epsilon^{3}\right), \\
C_{R, i}^{(0)}(\hat{r}, \hat{z}) & =\epsilon \frac{I_{s s}(\beta)}{2 \pi \mathcal{D} \hat{\rho}}+O\left(\epsilon^{3}\right)
\end{aligned}
$$

where $\hat{\rho}=\sqrt{\hat{r}^{2}+\hat{z}^{2}}$. Hence the leading-order terms $C_{O, o}^{(0)}$ and $C_{R, o}^{(0)}$ of the outer perturbation series, (32), are functions of $\hat{\rho}$ and $T$ only and are spherically symmetric, 
as the disk appears as a point source or sink on the outer length-scale; thus they satisfy the following time-dependent problems:

$$
\begin{aligned}
\frac{1}{\hat{\rho}} \frac{\partial^{2}}{\partial \hat{\rho}^{2}}\left(\hat{\rho} C_{O, o}^{(0)}\right) & =\frac{\partial C_{O, o}^{(0)}}{\partial T} & & z>0, \\
C_{O, o}^{(0)} & =0 & & T=0, \\
C_{O, o}^{(0)} & \rightarrow 0 & & \hat{\rho} \rightarrow \infty, \\
C_{O, o}^{(0)} & \sim-\frac{I_{s s}(\beta)}{2 \pi \hat{\rho}} & & \hat{\rho} \rightarrow 0,
\end{aligned}
$$

and

$$
\begin{aligned}
\frac{\mathcal{D}}{\hat{\rho}} \frac{\partial^{2}}{\partial \hat{\rho}^{2}}\left(\hat{\rho} C_{R, o}^{(0)}\right) & =\frac{\partial C_{R, o}^{(0)}}{\partial T} & & z>0, \\
C_{R, o}^{(0)} & =0 & & T=0, \\
C_{R, o}^{(0)} & \rightarrow 0 & & \hat{\rho} \rightarrow \infty, \\
C_{R, o}^{(0)} & \sim \frac{I_{s s}(\beta)}{2 \pi \mathcal{D} \hat{\rho}} & & \hat{\rho} \rightarrow 0,
\end{aligned}
$$

whose solutions are:

$$
\begin{aligned}
& C_{O, o}^{(0)}=-\frac{I_{s s}(\beta)}{2 \pi \hat{\rho}} \operatorname{erfc}\left(\frac{\hat{\rho}}{2 \sqrt{T}}\right) \\
& C_{R, o}^{(0)}=\frac{I_{s s}(\beta)}{2 \pi \mathcal{D} \hat{\rho}} \operatorname{erfc}\left(\frac{\hat{\rho}}{2 \sqrt{\mathcal{D} T}}\right) .
\end{aligned}
$$

\subsubsection{First-order inner solution}

Next we apply Van Dyke's matching rule [40] to determine the first-order influence of the outer solution upon the inner problem. Writing (39) and (40) in terms of the inner variable $\hat{\rho}=\epsilon \rho$, where $\rho=\sqrt{r^{2}+z^{2}}$, and taking the first two terms of the expansion as $\epsilon \rightarrow 0$, we obtain

$$
\begin{aligned}
& C_{O, o}=-\frac{I_{s s}(\beta)}{2 \pi \rho}+\epsilon \frac{I_{s s}(\beta)}{2 \pi^{\frac{3}{2}} \sqrt{T}}+O\left(\epsilon^{3}\right), \\
& C_{R, o}=\frac{I_{s s}(\beta)}{2 \pi \mathcal{D} \rho}-\epsilon \frac{I_{s s}(\beta)}{2 \pi^{\frac{3}{2}} \mathcal{D}^{\frac{3}{2}} \sqrt{T}}+O\left(\epsilon^{3}\right) .
\end{aligned}
$$

Hence, the first-order terms $C_{O, i}^{(1)}$ and $C_{R, i}^{(1)}$ of the inner perturbation series, (31a) and (31b), satisfy:

$$
\nabla^{2} C_{O, i}^{(1)}=0, \quad \nabla^{2} C_{R, i}^{(1)}=0,
$$

with boundary conditions as $\rho \rightarrow \infty$ :

$$
C_{O, i}^{(1)} \rightarrow \frac{I_{s s}(\beta)}{2 \pi^{\frac{3}{2}} \sqrt{T}}, \quad C_{R, i}^{(1)} \rightarrow-\frac{I_{s s}(\beta)}{2 \pi^{\frac{3}{2}} \mathcal{D}^{\frac{3}{2}} \sqrt{T}} .
$$


On the electrode surface, $r \leq 1, z=0$, the boundary conditions are:

$$
\begin{aligned}
\frac{\partial C_{O, i}^{(1)}}{\partial z} & =k_{f} C_{O, i}^{(1)}-k_{b} C_{R, i}^{(1)}, \\
\frac{\partial C_{O, i}^{(1)}}{\partial z} & =-\mathcal{D} \frac{\partial C_{R, i}^{(1)}}{\partial z} .
\end{aligned}
$$

For $r>1, z=0$, the no-flux condition is:

$$
\frac{\partial C_{O, i}^{(1)}}{\partial z}=\frac{\partial C_{R, i}^{(1)}}{\partial z}=0 .
$$

Using the governing equations, (43a), and the boundary conditions, (43b), (43d)-(43e), we see that the following quantity must be conserved:

$$
C_{O, i}^{(1)}+\mathcal{D} C_{R, i}^{(1)} \equiv-\frac{I_{s s}(\beta)}{2 \pi^{\frac{3}{2}} \sqrt{T}}\left(\mathcal{D}^{-\frac{1}{2}}-1\right)
$$

Hence $C_{R, i}^{(1)}$ can be eliminated from (43a)-(43e) to obtain a single problem for $C_{O, i}^{(1)}$ :

$$
\begin{array}{rlr}
\nabla^{2} C_{O, i}^{(1)} & =0 & z>0, \\
C_{O, i}^{(1)} \rightarrow \frac{I_{s s}(\beta)}{2 \pi^{\frac{3}{2}} \sqrt{T}} & \rho \rightarrow \infty, \\
-\frac{\partial C_{O, i}^{(1)}}{\partial z} & =\left\{\begin{array}{lll}
0 & r>1 & \\
\frac{k_{b} I_{s s}(\beta)}{2 \pi^{\frac{3}{2}} \mathcal{D} \sqrt{T}}\left(1-\mathcal{D}^{-\frac{1}{2}}\right)-\beta C_{O, i}^{(1)} & r \leq 1 & z=0 .
\end{array}\right.
\end{array}
$$

By comparison with (19), the solution to this problem can be written in terms of the steady-state solution, $C_{O}^{s s}$, as follows:

$$
C_{O, i}^{(1)}=\frac{I_{s s}(\beta)}{2 \pi^{\frac{3}{2}} \sqrt{T}}\left[1-\left(\frac{k_{f}+k_{b} \mathcal{D}^{-\frac{3}{2}}}{\beta}\right) C_{O}^{s s}(\beta ; r, z)\right] .
$$

From (44), we see that $C_{R, i}^{(1)}$ has the corresponding solution:

$$
C_{R, i}^{(1)}=-\frac{I_{s s}(\beta)}{2 \pi^{\frac{3}{2}} \mathcal{D} \sqrt{T}}\left[\mathcal{D}^{-\frac{1}{2}}-\left(\frac{k_{f}+k_{b} \mathcal{D}^{-\frac{3}{2}}}{\beta}\right) C_{O}^{s s}(\beta ; r, z)\right]
$$

\subsection{Analytical expression for the long-time transient current}

Collecting the terms in the inner perturbation series for $C_{O, i}$, (33) and (46), the solution for $C_{O, i}$ is therefore given by

$$
\begin{array}{r}
C_{O, i}=C_{O}^{s s}(\beta ; r, z)+\frac{\epsilon}{\sqrt{T}} \frac{I_{s s}(\beta)}{2 \pi^{\frac{3}{2}}}\left[1-\frac{k_{f}+k_{b} \mathcal{D}^{-\frac{3}{2}}}{\beta} C_{O}^{s s}(\beta ; r, z)\right] \\
+O\left(\left(\frac{\epsilon}{\sqrt{T}}\right)^{3}\right),
\end{array}
$$


while the corresponding solution for $C_{R, i}$ is found from (34) and (47) to be:

$$
\begin{aligned}
& C_{R, i}=-\frac{1}{\mathcal{D}} C_{O}^{s s}(\beta ; r, z)-\frac{\epsilon}{\sqrt{T}} \frac{I_{s s}(\beta)}{2 \pi^{\frac{3}{2}} \mathcal{D}}\left[\mathcal{D}^{-\frac{1}{2}}-\left(\frac{k_{f}+k_{b} \mathcal{D}^{-\frac{3}{2}}}{\beta}\right) C_{O}^{s s}(\beta ; r, z)\right] \\
& +O\left(\left(\frac{\epsilon}{\sqrt{T}}\right)^{3}\right) \text {. }
\end{aligned}
$$

In expressions (48) and (49), we have indicated the error term of $O\left((\epsilon / \sqrt{T})^{3}\right)$. In other words, it transpires that the second-order corrections of $O\left(\epsilon^{2} / T\right)$ are identically zero. We relegate the detailed justification of this to Appendix B.

Expression (48) implies that the long-time transient current is given by

$$
\begin{aligned}
I(t) & =\left.2 \pi \int_{0}^{1} \frac{\partial C_{O, i}}{\partial z}\right|_{z=0} r \mathrm{~d} r \\
& =I_{s s}(\beta)\left[1-\frac{I_{s s}(\beta)}{2 \pi^{\frac{3}{2}} \sqrt{t}}\left(\frac{k_{f}+k_{b} \mathcal{D}^{-\frac{3}{2}}}{\beta}\right)\right]+O\left(t^{-\frac{3}{2}}\right),
\end{aligned}
$$

where $I_{s s}(\beta)$ is the steady-state current defined by (21) and we return to the physical time variable $t=T / \epsilon^{2}$. Converting back to dimensional variables gives the main result of this article:

$$
\begin{aligned}
& \tilde{I}(\tilde{t}) \sim n F \tilde{a} \tilde{D}_{O} \tilde{D}_{R} I_{s s}(\beta)\left(\frac{\tilde{k}_{f} \tilde{C}_{O}^{*}-\tilde{k}_{b} \tilde{C}_{R}^{*}}{\tilde{k}_{f} \tilde{D}_{R}+\tilde{k}_{b} \tilde{D}_{O}}\right) \times \\
& {\left[1-\frac{\tilde{a} I_{s s}(\beta)}{2 \pi^{\frac{3}{2}} \sqrt{\tilde{t} \tilde{D}_{O} \tilde{D}_{R}}}\left(\frac{\tilde{k}_{f} \tilde{D}_{R}^{\frac{3}{2}}+\tilde{k}_{b} \tilde{D}_{O}^{\frac{3}{2}}}{\tilde{k}_{f} \tilde{D}_{R}+\tilde{k}_{b} \tilde{D}_{O}}\right)\right], \quad \text { as } \tilde{t} \rightarrow \infty, }
\end{aligned}
$$

where the error in the formula is proportional to $\tilde{t}^{-3 / 2}$ and $\beta$ is defined as in (2).

\section{Results and discussion}

In this section, we consider special cases of the solution for the current response (52) and show that it encapsulates existing solutions in the literature for diffusionlimited currents. We also verify the analytical solution by comparison with numerically calculated currents.

\subsection{Special cases of the current response}

\subsubsection{Extreme polarisation currents}

For a reduction reaction, extreme polarisation corresponds to letting $k_{f} \rightarrow \infty$ and $k_{b} \rightarrow 0$. Since $I_{s s}(\infty)=-4$, the resulting time-dependent limiting current is given by:

$$
\tilde{I}(\tilde{t}) \sim-4 n F \tilde{a} \tilde{D}_{O} \tilde{C}_{O}^{*}\left[1+\frac{2 \tilde{a}}{\pi^{\frac{3}{2}} \sqrt{\tilde{t} \tilde{D}_{O}}}\right] .
$$


This result agrees with the first two terms of the series reported by Shoup and Szabo [19] and Phillips [20]. Similarly for an oxidation reaction, $k_{b} \rightarrow \infty$ and $k_{f} \rightarrow 0$, so that the limiting current is given by:

$$
\tilde{I}(\tilde{t}) \sim 4 n F \tilde{a} \tilde{D}_{R} \tilde{C}_{R}^{*}\left[1+\frac{2 \tilde{a}}{\pi^{\frac{3}{2}} \sqrt{\tilde{t} \tilde{D}_{R}}}\right] .
$$

\subsubsection{Reversible reactions}

Aoki and Osteryoung [17, 18] (corrected by Shoup and Szabo [19]) found the complete expansion using the Wiener-Hopf method for the special case when $\tilde{C}_{R}^{*}=0$, $\tilde{D}_{O}=\tilde{D}_{R}=\tilde{D}$ and $k_{f}, k_{b} \rightarrow \infty$ such that $k_{b} / k_{f}=O(1)$. Rajendran and Sangaranarayanan [23] also reported four terms of the series for the current. In this case, $I_{s s}(\beta)=-4$, and, making the same assumptions in (52), we obtain the same result as the first two terms in their series, namely

$$
\tilde{I}(\tilde{t}) \sim-4 n F \tilde{a} \tilde{D} \tilde{C}_{O}^{*}\left(1+\frac{\tilde{k}_{b}}{\tilde{k}_{f}}\right)^{-1}\left[1+\frac{2 \tilde{a}}{\pi^{\frac{3}{2}} \sqrt{\tilde{D} \tilde{t}}}\right] .
$$

If the diffusion coefficients are not the same and $\tilde{C}_{R}^{*} \neq 0$, then the generalised result for reversible reactions is given by

$$
\begin{aligned}
\tilde{I}(\tilde{t}) \sim-4 n F \tilde{a} \tilde{D}_{O} \tilde{D}_{R}\left(\frac{\tilde{k}_{f} \tilde{C}_{O}^{*}-\tilde{k}_{b} \tilde{C}_{R}^{*}}{\tilde{k}_{f} \tilde{D}_{R}+\tilde{k}_{b} \tilde{D}_{O}}\right) \times & \\
& {\left[1+\frac{2 \tilde{a}}{\pi^{\frac{3}{2}} \sqrt{\tilde{t} \tilde{D}_{O} \tilde{D}_{R}}}\left(\frac{\tilde{k}_{f} \tilde{D}_{R}^{\frac{3}{2}}+\tilde{k}_{b} \tilde{D}_{O}^{\frac{3}{2}}}{\tilde{k}_{f} \tilde{D}_{R}+\tilde{k}_{b} \tilde{D}_{O}}\right)\right] . }
\end{aligned}
$$

\subsubsection{Irreversible reactions}

For an irreversible reduction reaction, $k_{b} \rightarrow 0$, whilst $k_{f}$ remains $O(1)$, so that

$$
\tilde{I}(\tilde{t}) \sim n F \tilde{a} \tilde{D}_{O} I_{s s}\left(\frac{\tilde{k}_{f} \tilde{a}}{\tilde{D}_{O}}\right) \tilde{C}_{O}^{*}\left[1-\frac{\tilde{a} I_{s s}\left(\frac{\tilde{k}_{f} \tilde{a}}{\tilde{D}_{O}}\right)}{2 \pi^{\frac{3}{2}} \sqrt{\tilde{t} \tilde{D}_{O}}}\right] .
$$

For an irreversible oxidation reaction, $k_{f} \rightarrow 0$, whilst $k_{b}$ remains $O(1)$, so that

$$
\tilde{I}(\tilde{t}) \sim-n F \tilde{a} \tilde{D}_{R} I_{s s}\left(\frac{\tilde{k}_{b} \tilde{a}}{\tilde{D}_{R}}\right) \tilde{C}_{R}^{*}\left[1-\frac{\tilde{a} I_{s s}\left(\frac{\tilde{k}_{b} \tilde{a}}{\tilde{D}_{R}}\right)}{2 \pi^{\frac{3}{2}} \sqrt{\tilde{t} \tilde{D}_{R}}}\right] .
$$

\subsection{Comparison with numerical simulations}

To verify our prediction (51) for the long-time current response, we performed numerical simulations using the fully implicit finite-difference (FIFD) method detailed by Gavaghan [26, 27], with a spatial mesh expanding exponentially from the edge of the disk. The problem to be solved is given by the governing equations (12), with 
initial conditions (13), far-field boundary conditions (14) implemented at the edge of the finite domain, and boundary conditions on the electrode (15) and the surrounding insulator (16).

For the dimensionless problem, we chose the region of integration to be $(0 \leq r \leq$ $\left.101=r_{\max }, \quad 0 \leq z \leq 100=z_{\max }\right)$ and solved for $0 \leq t \leq 10=t_{\max }$. Note that the domain of integration was larger than the $6 \sqrt{D t_{\max }}$ condition recommended by Britz, [41], to ensure that the finite boundaries have no effect on the processes at the electrode; in this case, $r_{\max }, z_{\max }$ must be greater than $\max \left(6 \sqrt{t_{\max }}, 6 \sqrt{\mathcal{D} t_{\max }}\right)$. Following Gavaghan [26, 27], we chose the mesh parameters to be $h_{\text {last }}=8 \times 10^{-5}$ and $f=1.175$, and the time-stepping was chosen to ensure accurate solutions at all times. The initial time-step was taken to be $10^{-6}$ and was increased by a factor of 10 after every 1000 steps. To test whether this was sufficiently accurate, we also performed the simulations with an initial time-step of $10^{-5}$ and found that there was a negligible difference in the values of the current over the entire time domain; the maximum percentage difference was less than $0.5 \%$ for all the simulations run.

In Figure 3, we show comparisons of the numerical and analytical solutions for various combinations of the parameters $k_{f}, k_{b}$ and $\mathcal{D}$. We have plotted $I(t) / \beta$ against $t$ to ensure that the full effect of different diffusion coefficients on the current is captured, since the non-dimensionalisation of the concentrations includes a factor of $1 / \beta$, cf. expression (10b). The percentage differences between the numerical and analytical solutions are plotted in Figure 4, which confirms that the analytical solution diverges from the numerical solution for small $t$ and converges for large $t$. We expect that the analytical solution (51) is valid for long-times $t$ such that

$$
t \gg \max \left(1, \mathcal{D}^{-1}\right)
$$

which is the non-dimensional equivalent of condition (30). For the parameters considered in Figure 4, the percentage difference between the analytical and numerical solutions is less than $1.5 \%$ for $t \geq 1$.

\section{Conclusions}

We have derived a novel approximate solution (52) for the long-time-dependent chronoamperometric current at a circular disk electrode. The solution generalises previous results in the literature to allow for quasi-reversible reactions at the electrode. It also extends the previous work to allow the oxidant and the reductant to have different diffusion coefficients. We showed that our new solution encapsulates and generalises the known solutions for diffusion-limited currents, and agrees well with numerically calculated solutions. Our analysis shows that the large-time current decays to its steady-state value like $\tilde{t}^{-1 / 2}$ as $\tilde{t} \rightarrow \infty$. A key conclusion of our work is that the correction of $O\left(\tilde{t}^{-1}\right)$ is identically zero, so that a simple two-term approximation gives surprisingly accurate results.

We have made no assumptions in this article about the form of the forward and backward rate constants, $\tilde{k}_{f}$ and $\tilde{k}_{b}$, other than that they are constant. The most commonly used model for the forward and backward rate constants is the Butler-Volmer model [42], which relates the rate constants to the applied potential at the electrode 
surface. In the future, we plan to discuss how the results in this paper can be applied to define a protocol for estimating the parameters of the Butler-Volmer model from a series of chronoamperometric experiments, and we will verify the protocol experimentally.

\section{Acknowledgements}

Funding for this project was provided by the Engineering and Physical Sciences Research Council (EPSRC) (grant number EP/F044690/1) and is gratefully acknowledged.

\section{Supplementary Data}

We supply a working curve for the non-dimensional steady-state current $I_{s s}(\beta)$ as a function of $\beta$ for the range $0 \leq \beta \leq 500$, at the points $\beta_{j}=0.05 \times(j-1)$, $j=1, \ldots, 10,001$. Details of its calculation are described in Appendix A. The file is called 'Iss_working_curve.txt'.

\section{References}

[1] K. Aoki, Theory of ultramicroelectrodes, Electroanalysis 5 (1993) 627-639.

[2] C. Amatore, Electrochemistry at ultramicroelectrodes, in: I. Rubinstein (Ed.), Physical Electrochemistry: Principles, Methods and Applications, Marcel Dekker, New York, 1995, pp. 131-208.

[3] C.A. Basha, L. Rajendran, Theories of ultramicrodisc electrodes: review article, Int. J. Electrochem. Sci. 1 (2006) 268-282.

[4] A.M. Bond, K.B. Oldham, C.G. Zoski, Theory of electrochemical processes at an inlaid disc microelectrode under steady-state conditions, J. Electroanal. Chem. 245 (1988) 71-104.

[5] J. Newman, Resistance for flow of current to a disk, J. Electrochem. Soc. 113 (1966) 501-502.

[6] Y. Saito, A theoretical study on the diffusion current at the stationary electrodes of circular and narrow band types, Rev. Polarography 15 (1968) 177-187.

[7] I.N. Sneddon, Mixed Boundary Value Problems in Potential Theory, first ed., John Wiley \& Sons Inc., New York, 1966.

[8] K.B. Oldham, Steady-state concentrations and fluxes in the vicinity of a reversible inlaid disc microelectrode, J. Electroanal. Chem. 260 (1989) 461-467.

[9] C.G. Phillips, The steady-state current for a microelectrode near diffusion-limited conditions, J. Electroanal. Chem. 291 (1990) 251-256. 
[10] M.A. Bender, H.A. Stone, An integral equation approach to the study of the steady state current at surface microelectrodes, J. Electroanal. Chem. 351 (1993) 29-55.

[11] K. Aoki, K. Tokuda, H. Matsuda, Theory of stationary current-potential curves at microdisk electrodes for quasi-reversible and totally irreversible electrode reactions, J. Electroanal. Chem. 235 (1987) 87-96.

[12] M. Fleischmann, J. Daschbach, S. Pons, The behavior of microdisk and microring electrodes. Application of Neumann's integral theorem to the prediction of the steady state response of microdisks, J. Electroanal. Chem. 263 (1989) 189-203.

[13] J. Daschbach, S. Pons, M. Fleischmann, The behavior of microdisk and microring electrodes. Application of Neumann's integral theorem to the prediction of the steady state response of microdisks. Numerical illustrations, J. Electroanal. Chem. 263 (1989) 205-224.

[14] D.R. Baker, M.W. Verbrugge, An integral-transform formulation for the reaction distribution on a stationary-disk electrode below the limiting current, J. Electrochem. Soc. 137 (1990) 1832-1842.

[15] D.R. Baker, M.W. Verbrugge, An analytic solution for the microdisk electrode and its use in the evaluation of charge-transfer rate constants, J. Electrochem. Soc. 137 (1990) 3836-3845.

[16] K.B. Oldham, C.G. Zoski, Steady-state voltammetry at an inlaid microdisc: comparison of three approaches, J. Electroanal. Chem. 313 (1991) 17-28.

[17] K. Aoki, J. Osteryoung, Diffusion-controlled current at the stationary finite disk electrode. Theory, J. Electroanal. Chem. 122 (1981) 19-35.

[18] K. Aoki, J. Osteryoung, Formulation of the diffusion-controlled current at very small stationary disk electrodes, J. Electroanal. Chem. 160 (1984) 335-339.

[19] D. Shoup, A. Szabo, Chronoamperometric current at finite disk electrodes, J. Electroanal. Chem. 140 (1982) 237-245.

[20] C.G. Phillips, The long-time transient of two- and three-dimensional diffusion in microelectrode chronoamperometry, J. Electroanal. Chem. 333 (1992) 11-32.

[21] C.G. Phillips, K.M. Jansons, The short-time transient of diffusion outside a conducting body, Proc. Roy. Soc. A 428 (1990) 431-449.

[22] K.B. Oldham, Edge effects in semiinfinite diffusion, J. Electroanal. Chem. 122 (1981) 1-17.

[23] L. Rajendran, M.V. Sangaranarayanan, Diffusion at ultramicro disk electrodes: chronoamperometric current for steady-state $\mathrm{Ec}^{\prime}$ reaction using scattering analogue techniques, J. Phys. Chem. B 103 (1999) 1518-1524. 
[24] M.Fleischmann, J. Daschbach, S. Pons, The behavior of microdisk and microring electrodes. Mass transport to the disk in the unsteady state. Chronoamperometry, J. Electroanal. Chem. 250 (1988) 269-276.

[25] M. Fleischmann, D. Pletcher, G. Denault, J. Daschbach, S. Pons, The behavior of microdisk and microring electrodes. Prediction of the chronoamperometric response of microdisks and of the steady state for CE and EC catalytic reactions by application of Neumann's integral theorem, J. Electroanal. Chem. 263 (1989) 225-236.

[26] D.J. Gavaghan, An exponentially expanding mesh ideally suited to the fast and efficient simulation of diffusion processes at microdisc electrodes. 1. Derivation of the mesh, J. Electroanal. Chem. 456 (1998) 1-12.

[27] D.J. Gavaghan, An exponentially expanding mesh ideally suited to the fast and efficient simulation of diffusion processes at microdisc electrodes. 2. Application to chronoamperometry, J. Electroanal. Chem. 456 (1998) 13-23.

[28] K. Harriman, D.J. Gavaghan, P. Houston, E. Süli, Adaptative finite element simulation of currents at microelectrodes to a guaranteed accuracy. Application to a simple model problem, Electrochem. Comm. 2 (2000) 150-156.

[29] K. Harriman, D.J. Gavaghan, P. Houston, E. Süli, Adaptive finite element simulation of currents at microelectrodes to a guaranteed accuracy. Theory, Electrochem. Comm. 2 (2000) 157-162.

[30] K. Harriman, D.J. Gavaghan, E. Süli, Adaptive finite element simulation of chronoamperometry at microdisc electrodes, Electrochem. Comm. 5 (2003) 519529 .

[31] A. Oleinick, C. Amatore, I. Svir, Efficient quasi-conformal map for simulation of diffusion at disk microelectrodes, Electrochem. Comm. 6 (2004) 588-594.

[32] C. Amatore, A.I. Oleinick, I. Svir, Construction of optimal quasi-conformal mappings for the 2D-numerical simulation of diffusion at microelectrodes. Part 1: Principle of the method and its application to the inlaid disk microelectrode, J. Electroanal. Chem. 597 (2006) 69-76.

[33] C. Amatore, A.I. Oleinick, I. Svir, Construction of optimal quasi-conformal mappings for the 2D numerical simulation of diffusion at microelectrodes. Part 2. Application to recessed or protruding electrodes and their arrays, J. Electroanal. Chem. 597 (2006) 77-85.

[34] C. Amatore, A.I. Oleinick, I. Svir, Theoretical analysis of microscopic ohmic drop effects on steady-state and transient voltammetry at the disk microelectrode: a quasi-conformal mapping modeling and simulation, Anal. Chem. 80 (2008) 7947-7956. 
[35] M.V. Mirkin, A.J. Bard, Multidimensional integral equations. Part 1. A new approach to solving microelectrode diffusion problems, J. Electroanal. Chem. 323 (1992) 1-27.

[36] C.J. Tranter, On some dual integral equations occurring in potential problems with axial symmetry, Quart. J. Mech. and Appl. Math. 4 (1950) 411-419.

[37] K. Nisanciog̈lu, J. Newman, The short-time response of a disk electrode, J. Electrochem. Soc. 121 (1974) 523-527.

[38] J. Newman, The Fundamental Principles of Current Distribution and Mass Transport in Electrochemical Cells, in: A. J. Bard (Ed.), Electroanalytical Chemistry, Vol. 6, Marcel Dekker Inc., New York, 1973, pp. 187-352.

[39] M. Abramowitz, I.A. Stegun, (Eds.) Handbook of Mathematical Functions with Formulas, Graphs, and Mathematical Tables, National Bureau of Standards Applied Mathematics Series, 55, U.S. Government Printing Office, Washington, DC, 1964.

[40] E.J. Hinch, Perturbation Methods, first ed., Cambridge University Press, Cambridge, 1991.

[41] D. Britz, Digital Simulation in Electrochemistry, second ed., Springer, Heidelberg, 1988.

[42] A.J. Bard, L.R. Faulkner, Electrochemical Methods: Fundamentals and Applications, second ed., John Wiley \& Sons, New York, 2001.

\section{Appendix A. Computing the steady-state non-dimensional current $I_{s s}(\beta)$}

To recap for clarity, the solution for the steady-state oxidant concentration, $C_{O}^{s s}(\beta ; r, z)$, satisfies the following problem (cf. (19)):

$$
\begin{aligned}
& \nabla^{2} C_{O}^{s s}=0 \quad z>0, \\
& C_{O}^{s s} \rightarrow 0 \quad z \rightarrow \infty, \\
& \frac{\partial C_{O}^{s s}}{\partial z}=q(\beta ; r)=\left\{\begin{array}{ll}
0 & r>1, \\
\beta\left(C_{O}^{s s}-1\right) & r \leq 1,
\end{array} \quad z=0 .\right.
\end{aligned}
$$

The non-dimensional steady-state current through the disk electrode is given by

$$
I_{s s}(\beta)=2 \pi \int_{0}^{1} \frac{\partial C_{O}^{s s}}{\partial z}(\beta ; r, 0) r \mathrm{~d} r=2 \pi \int_{0}^{1} q(\beta ; r) r \mathrm{~d} r .
$$

Let $\widehat{q}(\beta ; s)$ denote the Hankel transform of $q(\beta ; r)$, namely

$$
\widehat{q}(\beta ; s)=\int_{0}^{\infty} q(\beta ; r) J_{0}(r s) r \mathrm{~d} r .
$$


It is straightforward to deduce from (A.1) and (A.2) that $\widehat{q}(\beta ; s)$ must satisfy the dual integral equations

$$
\begin{cases}\int_{0}^{\infty} \widehat{q}(\beta ; s)(\beta+s) J_{0}(r s) \mathrm{d} s=-\beta & r \leq 1, \\ \int_{0}^{\infty} \widehat{q}(\beta ; s) J_{0}(r s) s \mathrm{~d} s=0 & r>1 .\end{cases}
$$

Once $\widehat{q}(\beta ; s)$ is determined, we can compute $I_{s s}(\beta)$ using

$$
I_{s s}(\beta)=2 \pi \widehat{q}(\beta ; 0) .
$$

We solve (A.4) to find $\widehat{q}(\beta ; s)$ using Tranter's method [36]. If we decompose $\widehat{q}(\beta ; s)$ into a series of the form

$$
\widehat{q}(\beta ; s)=\frac{1}{s} \sum_{n=0}^{\infty} a_{n} J_{2 n+1}(s),
$$

then (A.4b) is satisfied identically, while (A.4a) leads to an infinite system of linear algebraic equations for the coeffients $a_{n}$. We truncate the system at some large finite size $N$ and hence obtain a matrix equation of the form

$$
\sum_{n=0}^{N-1}\left(\delta_{m n}+\beta L_{m n}\right) a_{n}=-\beta \delta_{m 0},
$$

for $a_{0}, a_{1}, \cdots, a_{N-1}$, where

$$
L_{m n}=\frac{8(-1)^{m+n}(2 m+1)}{\pi(2 m+2 n+1)(2 m-2 n+1)(2 n-2 m+1)(2 m+2 n+3)},
$$

and $\delta_{m n}$ is the Kronecker delta. For each finite value of $\beta$ and $N$, (A.7) is easily inverted and the non-dimensional steady-state current is then recovered from

$$
I_{s s}^{(N)}(\beta)=\pi a_{0}
$$

Accurate computation of $I_{s s}(\beta)$ requires an estimate of the truncation error in $I_{s s}^{(N)}(\beta)$. Assuming that the error is proportional to $N^{-p}$ for some positive integer $p$, it is possible to determine that the relative error, $\operatorname{Err}_{N}(\beta)$, decreases as $N^{-6}$, where $\operatorname{Err}_{N}(\beta)$ is defined as:

$$
\operatorname{Err}_{N}(\beta)=\frac{\left|I_{s s}^{(N)}(\beta)-I_{s s}(\beta)\right|}{\left|I_{s s}(\beta)\right|} .
$$

We display a $\log$-log plot of $\operatorname{Err}_{N}(\beta)$ versus $N$ for $\beta=500$ in Figure A.1; the dashed line indicates the slope of -6 .

For practical purposes, it is useful to have a working curve for $I_{s s}(\beta)$. We have calculated a working curve for $I_{s s}(\beta)$ using $N=50$ for $0 \leq \beta \leq 500$ at the 
points $\beta=\beta_{j}$, where $\beta_{j}=0.05(j-1), j=1, \ldots, 10,001$. The curve is plotted in Figure 2 in the main text and is supplied as Supplementary Data in a file called

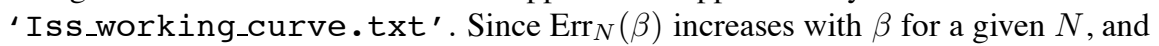
Figure A.1 shows that $\operatorname{Err}_{N}(500)=O\left(10^{-7}\right)$ at $N=50$, this implies that the relative error at each point on the calculated curve is less than $O\left(10^{-7}\right)$.

Finally, we note that the asymptotic approximation, (23), derived by Phillips [21] can be used to calculate $I_{s s}(\beta)$ for larger values of $\beta$; for $\beta>500$, the error is less than $0.2 \%$.

\section{Appendix B. Higher-order terms in the inner perturbation series solution (31)}

In the main body of the text we found the first two terms in the inner perturbation expansions for $C_{O, i}(r, z, T)$ and $C_{R, i}(r, z, T)$, (31). The leading-order solutions $C_{O, i}^{(0)}$ and $C_{R, i}^{(0)}$ are given by (33) and (34) respectively, while the first-order solutions $C_{O, i}^{(1)}$ and $C_{R, i}^{(1)}$ are given by (46) and (47). We have also found the leading-order terms in the outer perturbation expansions for $C_{O, o}(r, z, T)$ and $C_{R, o}(r, z, T),(32) ; C_{O, o}^{(0)}$ is given by (39) and $C_{R, o}^{(0)}$ is given by (40).

In this appendix, we continue the asymptotic matching procedure to find the firstorder outer solutions, $C_{O, o}^{(1)}$ and $C_{R, o}^{(1)}$. Subsequent matching back to the inner solution shows that the second-order inner solutions $C_{O, i}^{(2)}$ and $C_{R, i}^{(2)}$ are zero. This implies that the error in the inner perturbation expansions truncated at two terms is $O\left((\epsilon / \sqrt{T})^{3}\right)$ (or equivalently $O\left(t^{-3 / 2}\right)$ ), as we have indicated in expressions (48), (49) and (51).

\section{Appendix B.1. First-order outer solution}

Using the method of Van Dyke and the far-field behaviour of $C_{O}^{s s}(\beta ; r, z)$ given by (27), we find that the two-term outer expansion of the two-term inner solution is given by:

$$
\begin{gathered}
C_{O, i}=-\epsilon \frac{I_{s s}(\beta)}{2 \pi}\left(\frac{1}{\hat{\rho}}-\frac{1}{\sqrt{\pi T}}\right)+\epsilon^{2}\left(k_{f}+k_{b} \mathcal{D}^{-\frac{3}{2}}\right) \frac{I_{s s}(\beta)^{2}}{4 \pi^{\frac{5}{2}} \beta \sqrt{T} \hat{\rho}}+O\left(\epsilon^{3}\right) \\
C_{R, i}=\epsilon \frac{I_{s s}(\beta)}{2 \pi \mathcal{D}}\left(\frac{1}{\hat{\rho}}-\left(\frac{1}{\mathcal{D} \pi T}\right)^{\frac{1}{2}}\right) \\
\quad-\epsilon^{2}\left(k_{f}+k_{b} \mathcal{D}^{-\frac{3}{2}}\right) \frac{I_{s s}(\beta)^{2}}{4 \pi^{\frac{5}{2}} \mathcal{D} \beta \sqrt{T} \hat{\rho}}+O\left(\epsilon^{3}\right)
\end{gathered}
$$


Hence the outer solutions, $C_{O, o}^{(1)}$ and $C_{R, o}^{(1)}$, must satisfy the following problems:

$$
\begin{array}{rlrl}
\frac{1}{\hat{\rho}} \frac{\partial^{2}}{\partial \hat{\rho}^{2}}\left(\hat{\rho} C_{O, o}^{(1)}\right) & =\frac{\partial C_{O, o}^{(1)}}{\partial T} & & z>0, \\
C_{O, o}^{(1)} & =0 & T & =0, \\
C_{O, o}^{(1)} & \rightarrow 0 & \hat{\rho} \rightarrow \infty, \\
C_{O, o}^{(1)} & \sim\left(k_{f}+k_{b} \mathcal{D}^{-\frac{3}{2}}\right) \frac{I_{s s}(\beta)^{2}}{4 \pi^{\frac{5}{2}} \beta \sqrt{T} \hat{\rho}} & & \hat{\rho} \rightarrow 0,
\end{array}
$$

and

$$
\begin{aligned}
\frac{\mathcal{D}}{\hat{\rho}} \frac{\partial^{2}}{\partial \hat{\rho}^{2}}\left(\hat{\rho} C_{R, o}^{(1)}\right) & =\frac{\partial C_{R, o}^{(1)}}{\partial T} & & z>0, \\
C_{R, o}^{(1)} & =0 & & T=0, \\
C_{R, o}^{(1)} & \rightarrow 0 & & \hat{\rho} \rightarrow \infty, \\
C_{R, o}^{(1)} & \sim-\left(k_{f}+k_{b} \mathcal{D}^{-\frac{3}{2}}\right) \frac{I_{s s}(\beta)^{2}}{4 \pi^{\frac{5}{2}} \mathcal{D} \beta \sqrt{T} \hat{\rho}} & & \hat{\rho} \rightarrow 0,
\end{aligned}
$$

and the solutions are found to be:

$$
\begin{aligned}
C_{O, o}^{(1)} & =\left(k_{f}+k_{b} \mathcal{D}^{-\frac{3}{2}}\right) \frac{I_{s s}(\beta)^{2}}{4 \pi^{\frac{5}{2}} \beta \sqrt{T} \hat{\rho}} e^{-\frac{\hat{\rho}^{2}}{4 T}} \\
C_{R, o}^{(1)} & =-\left(k_{f}+k_{b} \mathcal{D}^{-\frac{3}{2}}\right) \frac{I_{s s}(\beta)^{2}}{4 \pi^{\frac{5}{2}} \mathcal{D} \beta \sqrt{T} \hat{\rho}} e^{-\frac{\hat{\rho}^{2}}{4 \mathcal{D} T}} .
\end{aligned}
$$

Appendix B.2. Second-order inner solution

The three-term inner expansion of the two-term outer solution is given by:

$$
\begin{aligned}
C_{O, o}=-\frac{I_{s s}(\beta)}{2 \pi \rho} & +\epsilon \frac{I_{s s}(\beta)}{2 \pi^{\frac{3}{2}} \sqrt{T}}\left(1+\left(k_{f}+k_{b} \mathcal{D}^{-\frac{3}{2}}\right) \frac{I_{s s}(\beta)}{2 \pi \beta \rho}\right) \\
& -\epsilon^{3} \frac{I_{s s}(\beta)}{2(\pi T)^{\frac{3}{2}}}\left[\frac{\rho^{2}}{12}+\left(k_{f}+k_{b} \mathcal{D}^{-\frac{3}{2}}\right) \frac{I_{s s}(\beta) \rho}{8 \pi \beta}\right]+O\left(\epsilon^{5}\right),
\end{aligned}
$$

and

$$
\begin{aligned}
C_{R, o}= & \frac{I_{s s}(\beta)}{2 \pi \mathcal{D} \rho}-\epsilon \frac{I_{s s}(\beta)}{2 \pi^{\frac{3}{2}} \mathcal{D} \sqrt{T}}\left(\mathcal{D}^{-\frac{1}{2}}+\left(k_{f}+k_{b} \mathcal{D}^{-\frac{3}{2}}\right) \frac{I_{s s}(\beta)}{2 \pi \beta \rho}\right) \\
& +\epsilon^{3} \frac{I_{s s}(\beta)}{2 \mathcal{D}^{2}(\pi T)^{\frac{3}{2}}}\left[\mathcal{D}^{-\frac{1}{2}} \frac{\rho^{2}}{12}+\left(k_{f}+k_{b} \mathcal{D}^{-\frac{3}{2}}\right) \frac{I_{s s}(\beta) \rho}{8 \pi \beta}\right]+O\left(\epsilon^{5}\right) .
\end{aligned}
$$

Since the coefficient of $\epsilon^{2}$ is zero, this means that $C_{O, i}^{(2)}$ and $C_{R, i}^{(2)}$ must satisfy linear homogeneous boundary-value problems, whose solutions are $C_{O, i}^{(2)}=C_{R, i}^{(2)}=0$, and hence there is no term of $O\left(\epsilon^{2}\right)$ in the inner solution. This allows us to deduce that the error in the inner perturbation expansion truncated at two terms is $O\left((\epsilon / \sqrt{T})^{3}\right)$ (or equivalently $O\left(t^{-3 / 2}\right)$ ). 
Far-field, $\tilde{r}^{2}+\tilde{z}^{2} \rightarrow \infty$

Electrode, $\tilde{r} \leq \tilde{a}, \tilde{z}=0$

$\tilde{C}_{O} \rightarrow \tilde{C}_{O}^{*}, \quad \tilde{C}_{R} \rightarrow \tilde{C}_{R}^{*}$

$\mathrm{Ox}+n \mathrm{e} \underset{\tilde{k}_{b}}{\stackrel{\tilde{k}_{f}}{\rightleftharpoons}} \mathrm{Red}$

Governing equations, $\tilde{z}>0$

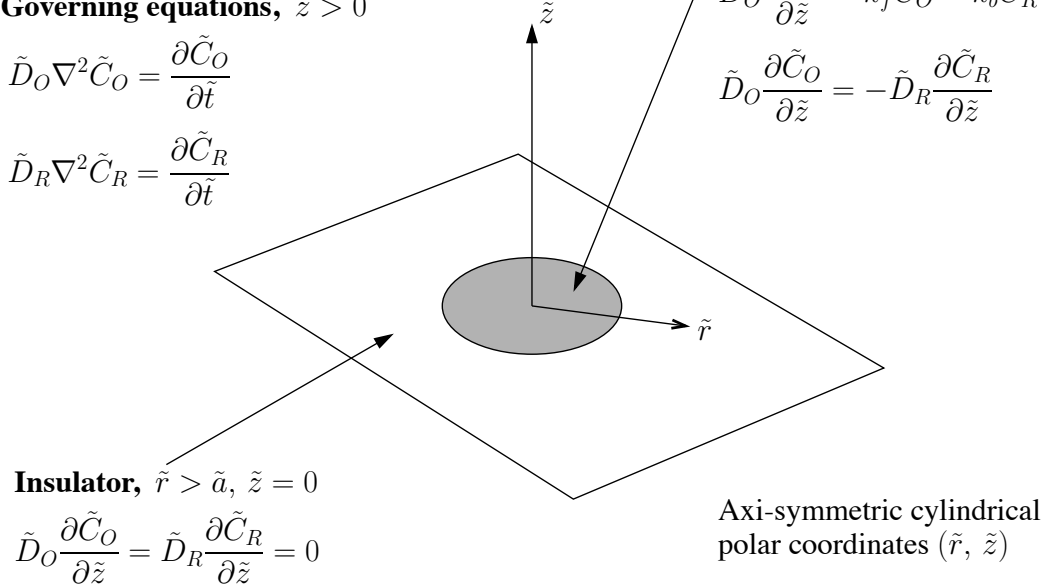

Figure 1: Schematic of the theoretical problem of an oxidant and reductant diffusing above a circular disk electrode of radius $\tilde{a}(\mathrm{~m})$ inlaid into an otherwise insulating plane at $\tilde{z}=0$. The concentration fields of the two species are denoted by $\tilde{C}_{O}(\tilde{r}, \tilde{z}, \tilde{t})$ and $\tilde{C}_{R}(\tilde{r}, \tilde{z}, \tilde{t})\left(\mathrm{mol} \mathrm{m}^{-3}\right)$ respectively, and their bulk concentrations in the far-field, $\tilde{C}_{O}^{*}$ and $\tilde{C}_{R}^{*}\left(\mathrm{~mol} \mathrm{~m}{ }^{-3}\right)$, are constant. Their diffusion coefficients are represented by $\tilde{D}_{O}$ and $\tilde{D}_{R}\left(\mathrm{~m}^{2} \mathrm{~s}^{-1}\right)$. A redox reaction with forward and backward reaction rates denoted by $\tilde{k}_{f}$ and $\tilde{k}_{b}$ $\left(\mathrm{m} s^{-1}\right)$ takes place at the electrode, where the two species exchange $n$ electrons.

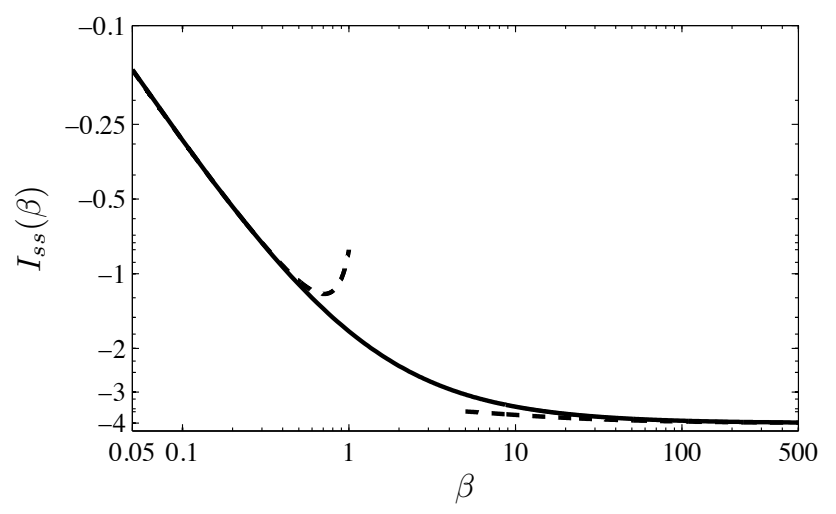

Figure 2: Log-log plot of the steady-state non-dimensional current, $I_{s s}(\beta)$, through a circular disk versus the mass transfer coefficient $\beta \in[0.05,500]$ (solid lines). The asymptotic approximations given by (22) as $\beta \rightarrow 0$ and (23) as $\beta \rightarrow \infty$ are shown as dashed curves. 


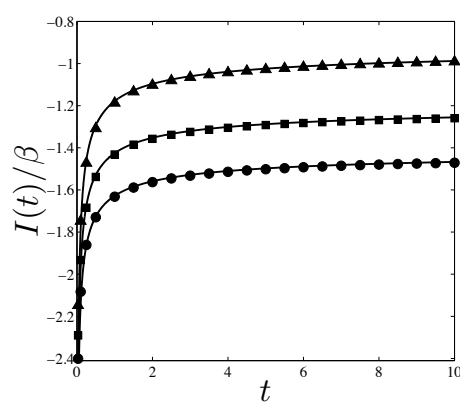

(a) Parameters $k_{f}=1, k_{b}=1$

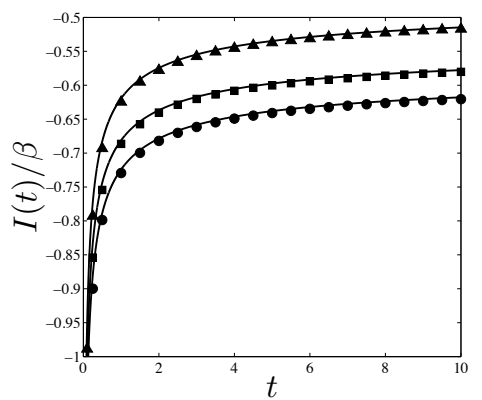

(b) Parameters $k_{f}=5, k_{b}=1$

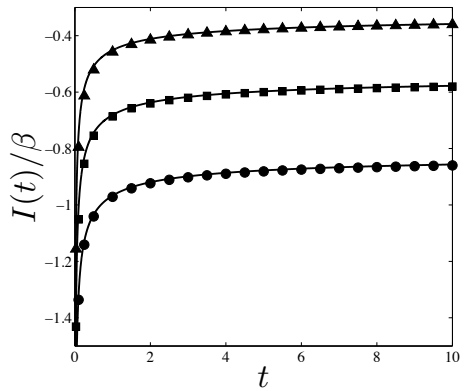

(c) Parameters $k_{f}=1, k_{b}=5$

Figure 3: Comparison between the non-dimensional analytical solution (solid lines) for the long-time transient current $I(t) / \beta$, where $I(t)$ is given by (51), with numerically simulated values (triangles, squares and circles) using the FIFD method devised by Gavaghan [26,27]. The three separate transients marked by the triangles, squares and circles on each plot correspond to taking $\mathcal{D}=0.5,1$, and 2 respectively and illustrate the impact of unequal diffusion coefficients. 


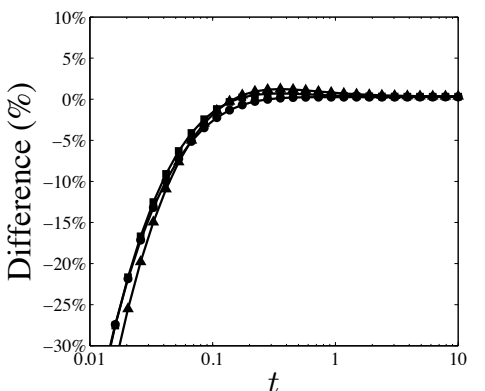

(a) Parameters $k_{f}=1, k_{b}=1$

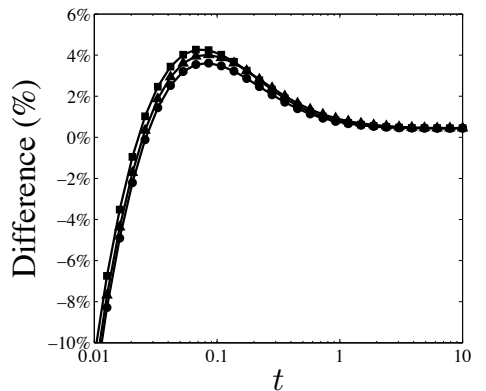

(b) Parameters $k_{f}=5, k_{b}=1$

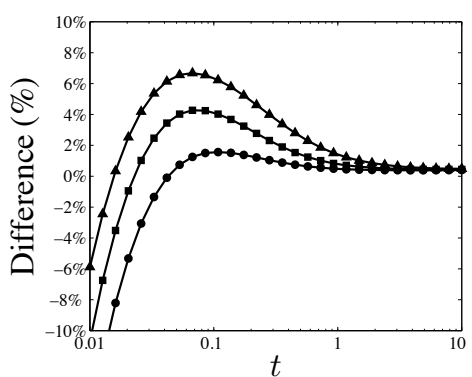

(c) Parameters $k_{f}=1, k_{b}=5$

Figure 4: Semi-log plots of the time-varying percentage difference between the non-dimensional long-time analytical solution for $I(t)$ given by (51) and numerically simulated values. The triangles, squares and circles denote different ratios of the diffusion coefficients, $\mathcal{D}=0.5,1,2$ respectively. As expected, the analytical solution diverges from the numerical solution at small times and converges at large times. For all the parameters considered, the percentage difference between the solutions is less than $1.5 \%$ for $t \geq 1$.

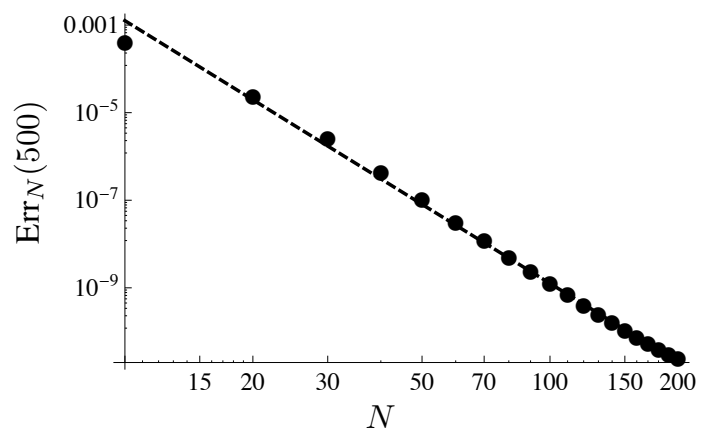

Figure A.1: Log-log plot of the relative error, $\operatorname{Err}_{N}(500)$, defined in (A.10), versus size $N$ of the truncated matrix equation (A.7). The dashed line indicates that the error decreases as $N^{-6}$. We note that choosing $N=50$ will ensure that the relative error in $I_{S s}^{(N)}(500)$ is $O\left(10^{-7}\right)$. 


\title{
The long-time chronoamperometric current at an inlaid disk electrode
}

\author{
Christopher G. Bell ${ }^{\mathrm{a}, 1, *}$, Peter D. Howell ${ }^{\mathrm{b}}$, Howard A. Stone ${ }^{\mathrm{c}}$, Wen-Jei Lim ${ }^{\mathrm{a}}$, Jennifer \\ H. Siggers ${ }^{\mathrm{a}}$ \\ ${ }^{a}$ Department of Bioengineering, Imperial College London, South Kensington Campus, London, SW7 2AZ, \\ $U K$ \\ ${ }^{b}$ Mathematical Institute, University of Oxford, 24-29 St Giles', Oxford, OX1 3LB, UK \\ ${ }^{c}$ Department of Mechanical and Aerospace Engineering, Princeton University, Princeton, New Jersey \\ 08544, United States
}

\begin{abstract}
Existing analytical solutions for the long-time chronoamperometric current response at an inlaid disk electrode are restricted to diffusion-limited currents due to extreme polarisation or reversible kinetics at the electrode surface. In this article, we derive an approximate analytical solution for the long-time-dependent current when the kinetics of the redox reaction at the electrode surface are quasi-reversible and the diffusion coefficients of the oxidant and reductant are different. We also detail a novel method for calculating the steady-state current. We show that our new method encapsulates and extends the existing solutions, and agrees with numerically simulated currents.
\end{abstract}

Keywords: chronoamperometry, disk, ultramicroelectrode, quasi-reversible, analytical

\section{Introduction}

Microdisk electrodes, and in particular ultra-microdisk electrodes, are popularly used for electrochemical investigations, since they possess many advantages [1-3]. A microdisk electrode is a conducting disk embedded in an insulating plane, and is easily fabricated by slicing through an insulated wire. Due to the geometry of the electrode, mass transport is enhanced at the edge of the disk, and the current scales with the radius of the disk rather than the area. The effects of ohmic drop and doublelayer capacitance are reduced, and the behaviour of electrochemical systems can be investigated over very small time- and length-scales. Miniaturisation of the electrode allows accurate information to be obtained about reactions with fast kinetics, which would be impossible to distinguish at larger electrodes, [4]. Since this type of electrode

\footnotetext{
*Corresponding author

Email address: bell@maths.ox.ac .uk (Christopher G. Bell)

${ }^{1}$ Present address: Mathematical Institute, University of Oxford, 24-29 St Giles', Oxford, OX1 3LB, UK, Tel: +44 (0)1865 273525, Fax: +44 (0)1865 273583
} 
is so widely used, theoretical research is vital to understand how the current response should behave. Theoretical investigations are complicated by the different boundary conditions on the electrode and the insulator, which results in a discontinuity in the flux normal to the surface at the electrode edge.

The general problem involves two redox species, Ox and Red, diffusing above a disk electrode with radius $\tilde{a}$ inlaid in an insulating plane. The diffusion coefficients for Ox and Red are denoted $\tilde{D}_{O}$ and $\tilde{D}_{R}$ respectively, and they are not generally equal. Provided the potential at the electrode is stepped to a constant value, the following redox reaction occurs at the electrode and produces a chronoamperometric current:

$$
\mathrm{Ox}+n \mathrm{e} \underset{\tilde{k}_{b}}{\stackrel{\tilde{k}_{f}}{\rightleftharpoons}} \operatorname{Red},
$$

where the forward and backward reaction rates, $\tilde{k}_{f}$ and $\tilde{k}_{b}$, are constant. If the effects of migration and natural convection can be neglected, then the current produced is a function of the rate of mass transport to the electrode due to diffusion and the rate of the reaction itself. Eventually the current reaches a steady state.

Analytically, investigations into the current produced at a disk electrode started with the steady-state problem. The earliest recorded solutions in the electrochemical literature date back to Newman [5] and Saito [6], who reported the formula for the diffusion-limited current due to extreme polarization, which was also well-known from potential theory [7]. For reversible kinetics, where the Nernst equation applies at the electrode surface, the analytical formula for the resulting diffusion-limited current is also well-known, cf. Bond et al. [4] (using the properties of discontinuous integrals of Bessel functions) and Oldham [8] (using spheroidal coordinates). More generally, reversible diffusion-limited currents occur whenever the following dimensionless parameter is infinite (cf. Phillips [9]):

$$
\beta=\frac{\tilde{k}_{f} \tilde{a}}{\tilde{D}_{O}}+\frac{\tilde{k}_{b} \tilde{a}}{\tilde{D}_{R}} .
$$

If $\beta$ is finite, then the reaction at the electrode is quasi-reversible. In this case, the steady-state current depends on a function of $\beta$, which generally must be calculated numerically. Analytical approximations have been derived by Phillips [9] for large $\beta$ (when the current is close to diffusion-limited), and by Bender and Stone [10] for small $\beta$. Bender and Stone [10] also used a Green's function approach to derive an integral equation for the current for any $\beta$, which they solved numerically. Aoki et al. [11] used the Wiener-Hopf method to show that the steady-state current for a quasireversible reaction can be calculated by solving a truncated infinite set of simultaneous equations. Three other approaches have been illustrated in the literature, namely that of Bond et al. [4] (using the properties of discontinuous integrals of Bessel functions), Fleischmann, Daschbach and Pons [12, 13] (using the Neumann integral theorem) and Baker and Verbrugge $[14,15]$ (using an integral equation written in terms of elliptic integrals, similar to the approach of Bender and Stone [10]). Oldham and Zoski [16] demonstrated that these three approaches are fundamentally similar and showed that they yield the same numerical values. 
The behaviour of the transient current before the system reaches steady-state, corresponding to a chronoamperometric experiment, is also of interest to researchers. For reversible reactions, and assuming that the diffusion coefficients of the oxidant and reductant are equal, Aoki and Osteryoung $[17,18]$ used the Wiener-Hopf procedure to develop approximate series expansions for the transient currents at short time and long time; the long-time series was subsequently corrected by Shoup and Szabo [19]. As part of a more general article on the long-time transient currents to microelectrodes of arbitrary shape, Phillips [20] showed that, in the special case of an inlaid disk, his solution agreed with Shoup and Szabo's correction. Due to an approximation in Aoki and Osteryoung's analysis [17], there was some doubt about the third term in the short-time series [18, 19], and Phillips and Jansons [21] derived a corrected version of the series. Oldham [22] found the first two terms in the short-time series for the diffusion-limited current in the case of extreme polarisation. Rajendran and Sangaranarayanan [23] also derived five- and four-term series respectively for the diffusionlimited currents at short- and long-time using results from scattering analogue theory, valid for equal diffusion coefficients. Fleischmann and coworkers also considered the chronoamperometric response of a disk electrode at extreme polarisation. In [24], they find an approximate solution in the Laplace-transformed variable, which satisfies the constant concentration boundary condition on average across the disk; and, in [25], they use Neumann's integral theorem to find a series solution (which they also extend to irreversible reactions), the time-dependent coefficients of which must be determined from a system of complicated equations.

A number of different numerical approaches have been developed to investigate both the steady-state current and the transient chronoamperometric current. Gavaghan $[26,27]$ developed a finite-difference approach using a spatial grid expanding exponentially from the electrode edge. Harriman, Gavaghan, Süli et al. [28-30] used an adaptive finite-element approach. Amatore, Oleinick and Svir [31-34] have described how to use quasi-conformal mapping techniques. Mirkin and Bard [35] showed how the transient current can be calculated from a multi-dimensional integral equation. Although extremely useful, these numerical simulations cannot provide the same direct insight as analytical solutions into how the current response depends on the underlying system parameters.

All of the analytical work on transient chronoamperometric currents descibed above only covers diffusion-limited currents, due to extreme polarisation or reversible kinetics, when the parameter $\beta$ is infinite. For the reversible kinetics/infinite- $\beta$ case, existing analysis also requires that the diffusion coefficients of the oxidant and the reductant are equal. In this article, we derive a two-term asymptotic series for the general long-time chronoamperometric current. For the reader who wishes to skip the detailed derivation, the final expression is given in equation (52). The solution extends the prior work described above to allow for quasi-reversible kinetics at the electrode and unequal diffusion coefficients. By 'long-time', we mean that the solution is valid for times, $\tilde{t}$, such that the following condition is satisfied:

$$
\tilde{t} \gg \max \left(\frac{\tilde{a}^{2}}{\tilde{D}_{O}}, \frac{\tilde{a}^{2}}{\tilde{D}_{R}}\right) .
$$

We demonstrate that the solution encapsulates the existing solutions for the diffusion- 
limited currents, and we show that it agrees with numerically simulated values using Gavaghan's finite-difference method [26, 27]. The first term in the series is the steadystate current, and the second term is proportional to $\tilde{t}^{-1 / 2}$, and depends on the square of the steady-state current. As detailed above, solutions for the steady-state current for a quasi-reversible reaction are already known. However, whilst carrying out this research, we found a new solution for the steady-state current using Tranter's method [36], which exploits the properties of discontinuous integrals of Bessel functions, and we report this in Appendix A. The approach is similar to Bond et al. [4], but uses different weighting functions. The resulting truncated infinite system of equations to be solved is easy to implement, since the coefficients in the matrix are simple, and converge quickly.

\section{Theory}

\subsection{Problem statement and non-dimensionalisation}

A schematic of the dimensional theoretical problem is displayed in Figure 1 (tildes indicate dimensional variables). We consider a simple redox reaction (1) between two species, Ox and Red, diffusing in the half-space $\tilde{z}>0$, which exchange $n$ electrons at disk electrode placed in the plane $\tilde{z}=0$. The forward and backward rate constants of the reaction are denoted by $\tilde{k}_{f}$ and $\tilde{k}_{b}$ respectively, and we will assume that the electrode is held at a constant potential so that they are both constant. The inlaid disk electrode has its centre situated at $\tilde{r}=0, \tilde{z}=0$ and has radius $\tilde{r}=\tilde{a}(\mathrm{~m})$. If any effects due to migration and convection are neglected, then the concentrations of Ox and Red, $\tilde{C}_{O}(\tilde{r}, \tilde{z}, \tilde{t})$ and $\tilde{C}_{R}(\tilde{r}, \tilde{z}, \tilde{t})$, each satisfy the diffusion equation for $\tilde{z}>0$ with constant diffusion coefficients $\tilde{D}_{O}$ and $\tilde{D}_{R}\left(\mathrm{~m}^{2} \mathrm{~s}^{-1}\right)$, that is:

$$
\tilde{D}_{O} \nabla^{2} \tilde{C}_{O}=\frac{\partial \tilde{C}_{O}}{\partial \tilde{t}}, \quad \tilde{D}_{R} \nabla^{2} \tilde{C}_{R}=\frac{\partial \tilde{C}_{R}}{\partial \tilde{t}} .
$$

Initially the bulk concentration of each species is constant everywhere:

$$
\tilde{C}_{O}(\tilde{r}, \tilde{z}, 0)=\tilde{C}_{O}^{*}, \quad \tilde{C}_{R}(\tilde{r}, \tilde{z}, 0)=\tilde{C}_{R}^{*} .
$$

We assume that the bulk concentrations remain undisturbed as the reaction at the electrode progresses, which provides the far-field boundary conditions

$$
\tilde{C}_{O} \rightarrow \tilde{C}_{O}^{*}, \quad \tilde{C}_{R} \rightarrow \tilde{C}_{R}^{*}, \quad \text { as } \tilde{r}^{2}+\tilde{z}^{2} \rightarrow \infty .
$$

On the electrode surface, the boundary conditions are given by the reaction at the surface and conservation of matter:

$$
\left.\begin{array}{l}
\tilde{D}_{O} \frac{\partial \tilde{C}_{O}}{\partial \tilde{z}}=\tilde{k}_{f} \tilde{C}_{O}-\tilde{k}_{b} \tilde{C}_{R}, \\
\tilde{D}_{O} \frac{\partial \tilde{C}_{O}}{\partial \tilde{z}}=-\tilde{D}_{R} \frac{\partial \tilde{C}_{R}}{\partial \tilde{z}},
\end{array}\right\} \quad \text { for } \tilde{r} \leq \tilde{a}, \tilde{z}=0
$$


There is no flux through the remainder of the surface, so that

$$
\tilde{D}_{O} \frac{\partial \tilde{C}_{O}}{\partial \tilde{z}}=\tilde{D}_{R} \frac{\partial \tilde{C}_{R}}{\partial \tilde{z}}=0, \text { for } \tilde{r}>\tilde{a}, \tilde{z}=0 .
$$

The Faradaic current through the electrode is given by

$$
\tilde{I}(\tilde{t})=-2 \pi n F \tilde{D}_{O} \int_{0}^{\tilde{a}} \frac{\partial \tilde{C}_{O}}{\partial \tilde{z}}(\tilde{r}, 0, \tilde{t}) \tilde{r} \mathrm{~d} \tilde{r}
$$

where $F$ is Faraday's constant, and we recall that $n$ is the number of electrons transferred in the redox reaction.

To non-dimensionalise the problem, we choose the following scalings:

$$
\begin{gathered}
\tilde{r}=\tilde{a} r, \quad \tilde{z}=\tilde{a} z, \quad \tilde{t}=\frac{\tilde{a}^{2}}{\tilde{D}_{O}} t, \quad \tilde{k}_{f}=\frac{\tilde{D}_{O}}{\tilde{a}} k_{f}, \quad \tilde{k}_{b}=\frac{\tilde{D}_{O}}{\tilde{a}} k_{b}, \\
\tilde{C}_{O}=\tilde{C}_{O}^{*}-\left(\frac{k_{f} \tilde{C}_{O}^{*}-k_{b} \tilde{C}_{R}^{*}}{k_{f}+k_{b} \mathcal{D}^{-1}}\right) C_{O}, \quad \tilde{C}_{R}=\tilde{C}_{R}^{*}-\left(\frac{k_{f} \tilde{C}_{O}^{*}-k_{b} \tilde{C}_{R}^{*}}{k_{f}+k_{b} \mathcal{D}^{-1}}\right) C_{R}, \\
\tilde{I}=n F \tilde{a} \tilde{D}_{O}\left(\frac{k_{f} \tilde{C}_{O}^{*}-k_{b} \tilde{C}_{R}^{*}}{k_{f}+k_{b} \mathcal{D}^{-1}}\right) I
\end{gathered}
$$

where

$$
\mathcal{D}=\frac{\tilde{D}_{R}}{\tilde{D}_{O}} .
$$

Then, in terms of the non-dimensional variables, the problem becomes:

$$
\nabla^{2} C_{O}=\frac{\partial C_{O}}{\partial t}, \quad \mathcal{D} \nabla^{2} C_{R}=\frac{\partial C_{R}}{\partial t}, \quad \text { in } z>0 .
$$

The initial conditions become:

$$
C_{O}(r, z, 0)=0, \quad C_{R}(r, z, 0)=0,
$$

and the far-field boundary conditions are

$$
C_{O} \rightarrow 0, \quad C_{R} \rightarrow 0, \quad \text { as } r^{2}+z^{2} \rightarrow \infty .
$$

On the electrode surface, the boundary conditions are:

$$
\left.\begin{array}{rl}
\frac{\partial C_{O}}{\partial z} & =\left(k_{f}+k_{b} \mathcal{D}^{-1}\right)\left(\frac{k_{f} C_{O}-k_{b} C_{R}}{k_{f}+k_{b} \mathcal{D}^{-1}}-1\right), \\
\frac{\partial C_{O}}{\partial z} & =-\mathcal{D} \frac{\partial C_{R}}{\partial z},
\end{array}\right\} \quad \text { for } r \leq 1, z=0 .
$$

On the remaining surface, the no-flux condition is:

$$
\frac{\partial C_{O}}{\partial z}=\frac{\partial C_{R}}{\partial z}=0, \text { for } r>1, z=0
$$

The dimensionless Faradaic current through the electrode is given by

$$
I(t)=2 \pi \int_{0}^{1} \frac{\partial C_{O}}{\partial z}(r, 0, t) r \mathrm{~d} r .
$$




\subsection{Steady-state problem}

The long-time solution of the time-dependent problem is highly reliant on the solution to the steady-state problem, which we consider here first. The steady-state concentrations, which we denote $C_{O}^{s s}$ and $C_{R}^{s s}$, satisfy Laplace's equation:

$$
\nabla^{2} C_{O}^{s s}=0, \quad \nabla^{2} C_{R}^{s s}=0
$$

in $z>0$, along with the boundary conditions (14)-(16). It is simple to see that $C_{O}^{s s}+$ $\mathcal{D} C_{R}^{s s}=0$ for all $r, z$, so that solution of the steady-state problem reduces to solving the following problem for $C_{O}^{s s}$ :

$$
\begin{array}{rlrl}
\nabla^{2} C_{O}^{s s} & =0 & & z>0, \\
C_{O}^{s s} & \rightarrow 0 & z \rightarrow \infty, \\
\frac{\partial C_{O}^{s s}}{\partial z}=q(\beta ; r) & = \begin{cases}0 & r>1, \\
\beta\left(C_{O}^{s s}-1\right) & r \leq 1,\end{cases} & z=0,
\end{array}
$$

where the mass transfer coefficient $\beta$ is given by

$$
\beta=k_{f}+k_{b} \mathcal{D}^{-1} \text {. }
$$

(This is expression (2) written in non-dimensional variables.) The solution to this problem, $C_{O}^{s s}=C_{O}^{s s}(\beta ; r, z)$, depends parametrically on the single parameter $\beta$, and the steady-state current, $I_{s s}(\beta)$, is given by (cf. (17))

$$
I_{s s}(\beta)=\left.2 \pi \int_{0}^{1} \frac{\partial C_{O}^{s s}}{\partial z}\right|_{z=0} r \mathrm{~d} r=2 \pi \int_{0}^{1} q(\beta ; r) r \mathrm{~d} r .
$$

The steady-state current as a function of $\beta$ can be computed in a number of ways as described in the Introduction [4, 10-16]. We have found a new solution using Tranter's method [36], which we detail in Appendix A. This methodology uses the properties of discontinuous integrals of Bessel functions, and is similar to that employed by Bond et al. [4], but uses different weighting functions. Our methodology results in a simpler matrix equation to solve for the current and it converges very quickly as the size of the matrix is increased. The result is shown as a log-log plot in Figure 2, along with the small- and large- $\beta$ asymptotes. The small- $\beta$ asymptote was derived by Bender and Stone [10], and is given by:

$$
I_{s s}(\beta) \sim-\pi \beta+\frac{8}{3} \beta^{2}-2.294 \beta^{3}+1.969 \beta^{4}+O\left(\beta^{5}\right), \quad \text { as } \beta \rightarrow 0,
$$

while the large- $\beta$ asymptote was derived by Phillips [9] to be:

$$
I_{s s}(\beta)=-4\left(1-(\pi \beta)^{-1} \log \beta+o\left(\beta^{-1} \log \beta\right)\right), \quad \text { as } \beta \rightarrow \infty .
$$

By comparing their numerical solution to this asymptotic approximation, Bender and Stone [10] suggested that (23) can be improved by adding a numerically based correction of $O\left(\beta^{-1}\right)$, so that the asymptote is given by:

$$
I_{s s}(\beta)=-4\left(1-\beta^{-1}\left(\pi^{-1} \log \beta+0.725\right)+o\left(\beta^{-1} \log \beta\right)\right), \quad \text { as } \beta \rightarrow \infty .
$$


Nisanciog̈lu and Newman [37] found the coefficient of the extra $O\left(\beta^{-1}\right)$ term to be 0.708 .

Using a Green's function, the solution for $C_{O}^{s s}$ can be expressed in terms of the flux, $q(\beta ; r)$, defined in $(19 \mathrm{c})$, as $[14,38]$ :

$$
C_{O}^{s s}(\beta ; r, z)=-\frac{2}{\pi} \int_{0}^{1} \mathrm{~K}\left(\frac{4 r s}{(r+s)^{2}+z^{2}}\right) \frac{q(\beta ; s) s \mathrm{~d} s}{\sqrt{(r+s)^{2}+z^{2}}}
$$

where $\mathrm{K}(m)$ denotes a complete elliptic integral of the first kind ([39], p. 590, 17.3.1):

$$
\mathrm{K}(m)=\int_{0}^{\pi / 2} \frac{1}{\sqrt{1-m \sin ^{2} \theta}} \mathrm{d} \theta .
$$

Solution of the transient problem requires knowledge of the far-field behaviour of $C_{O}^{s s}(\beta ; r, z)$, which can be derived from (25) to be:

$$
\begin{array}{r}
C_{O}^{s s}(\beta ; r, z) \sim-\frac{I_{s s}(\beta)}{2 \pi \sqrt{r^{2}+z^{2}}}-\frac{\left(r^{2}-2 z^{2}\right)}{4\left(r^{2}+z^{2}\right)^{5 / 2}} \int_{0}^{1} q(\beta ; s) s^{3} \mathrm{~d} s+\cdots, \\
\text { as } r^{2}+z^{2} \rightarrow \infty .
\end{array}
$$

To leading order, the far-field influence of the disk is characterised entirely by the steady-state current $I_{s s}(\beta)$ and is equivalent to a point source of strength $I_{s s}(\beta)$.

Since $I_{s s}(\beta)$ is critical for the understanding of the current response, we have supplied a working curve for $I_{s s}(\beta)$ as a function of $\beta$ over the range $0 \leq \beta \leq 500$ in the Supplementary Information; details of the calculation are set out in Appendix A. For $\beta>500$, the asymptotic approximations (23) or (24) for large $\beta$ can be used.

\subsection{Asymptotic solution for the long-time transient behaviour}

To find the long-time solution, we perform a coordinate expansion for large $t$ by letting

$$
t=\frac{T}{\epsilon^{2}}
$$

where $T=O(1)$ and $\epsilon \ll 1$, so that the governing equations (12) become:

$$
\nabla^{2} C_{O}=\epsilon^{2} \frac{\partial C_{O}}{\partial T}, \quad \mathcal{D} \nabla^{2} C_{R}=\epsilon^{2} \frac{\partial C_{R}}{\partial T} .
$$

In dimensional terms, the condition $\epsilon \ll 1$ is equivalent to assuming condition (3) mentioned in the Introduction, and which we repeat here:

$$
\tilde{t} \gg \max \left(\frac{\tilde{a}^{2}}{\tilde{D}_{O}}, \frac{\tilde{a}^{2}}{\tilde{D}_{R}}\right) .
$$

For example, for a microdisk electrode with radius $\tilde{a} \approx 10^{-5} \mathrm{~m}$ and diffusion coefficients $\tilde{D}_{O}, \tilde{D}_{R} \approx 10^{-10} \mathrm{~m}^{2} \mathrm{~s}^{-1}$, this condition implies that the solution that we derive will be valid for time-scales $\tilde{t} \gg 1 \mathrm{~s}$. 
Since $\epsilon \ll 1$, there is an inner region near the disk where the concentrations are at a steady state to leading order, and a outer region far from the disk where the concentrations are time-dependent to leading order. We find solutions in both regions using approximate matched asymptotic expansions. This approach is similar to that used for the diffusion-limited current by Phillips [20], who performed the analysis in the Laplace-transform domain. In the inner region, the coordinate system is simply $(r, z)$ as defined above, and we denote the inner dependent variables by using the subscript $i$, so that they are $C_{O, i}(r, z, T)$ and $C_{R, i}(r, z, T)$. In the outer region, we define the coordinate system to be $(\hat{r}, \hat{z})$, where $\hat{r}=\epsilon r$ and $\hat{z}=\epsilon z$, and we denote the dependent variables using the subscript $o: C_{O, o}(\hat{r}, \hat{z}, T)$ and $C_{R, o}(\hat{r}, \hat{z}, T)$.

We expand the inner variables in the following perturbation series:

$$
\begin{aligned}
& C_{O, i}(r, z, T)=C_{O, i}^{(0)}(r, z)+\epsilon C_{O, i}^{(1)}(r, z, T)+\epsilon^{2} C_{O, i}^{(2)}(r, z, T)+\ldots, \\
& C_{R, i}(r, z, T)=C_{R, i}^{(0)}(r, z)+\epsilon C_{R, i}^{(1)}(r, z, T)+\epsilon^{2} C_{R, i}^{(2)}(r, z, T)+\ldots,
\end{aligned}
$$

and the outer variables as:

$$
\begin{aligned}
& C_{O, o}(\hat{r}, \hat{z}, T)=\epsilon C_{O, o}^{(0)}(\hat{r}, \hat{z}, T)+\epsilon^{2} C_{O, o}^{(1)}(\hat{r}, \hat{z}, T)+\epsilon^{3} C_{O, o}^{(2)}(\hat{r}, \hat{z}, T)+\ldots, \\
& C_{R, o}(\hat{r}, \hat{z}, T)=\epsilon C_{R, o}^{(0)}(\hat{r}, \hat{z}, T)+\epsilon^{2} C_{R, o}^{(1)}(\hat{r}, \hat{z}, T)+\epsilon^{3} C_{R, o}^{(2)}(\hat{r}, \hat{z}, T)+\ldots
\end{aligned}
$$

\subsubsection{Leading-order inner solution}

To leading order, the concentrations are at steady state in the vicinity of the electrode, so that $C_{O, i}^{(0)}(r, z)$ and $C_{R, i}^{(0)}(r, z)$ satisfy the steady-state problem discussed in Section 2.2. Hence

$$
C_{O, i}^{(0)}(r, z)=C_{O}^{s s}(\beta ; r, z),
$$

where the steady-state solution, $C_{O}^{s s}$, is given by expression (25), and the corresponding leading-order solution for $C_{R, i}^{(0)}$ is:

$$
C_{R, i}^{(0)}(r, z)=-\frac{1}{\mathcal{D}} C_{O}^{s s}(\beta ; r, z) .
$$

\subsubsection{Leading-order outer solution}

Now we apply Van Dyke's matching rule [40].Writing the inner solutions $C_{O, i}^{(0)}$ and $C_{R, i}^{(0)}$ in terms of the outer variables $\hat{r}$ and $\hat{z}$, and letting $\epsilon$ tend to zero, we find from (27) that

$$
\begin{aligned}
& C_{O, i}^{(0)}(\hat{r}, \hat{z})=-\epsilon \frac{I_{s s}(\beta)}{2 \pi \hat{\rho}}+O\left(\epsilon^{3}\right), \\
& C_{R, i}^{(0)}(\hat{r}, \hat{z})=\epsilon \frac{I_{s s}(\beta)}{2 \pi \mathcal{D} \hat{\rho}}+O\left(\epsilon^{3}\right),
\end{aligned}
$$

where $\hat{\rho}=\sqrt{\hat{r}^{2}+\hat{z}^{2}}$. Hence the leading-order terms $C_{O, o}^{(0)}$ and $C_{R, o}^{(0)}$ of the outer perturbation series, (32), are functions of $\hat{\rho}$ and $T$ only and are spherically symmetric, 
as the disk appears as a point source or sink on the outer length-scale; thus they satisfy the following time-dependent problems:

$$
\begin{array}{rlrl}
\frac{1}{\hat{\rho}} \frac{\partial^{2}}{\partial \hat{\rho}^{2}}\left(\hat{\rho} C_{O, o}^{(0)}\right) & =\frac{\partial C_{O, o}^{(0)}}{\partial T} & & z>0, \\
C_{O, o}^{(0)} & =0 & & T=0, \\
C_{O, o}^{(0)} & \rightarrow 0 & \hat{\rho} \rightarrow \infty, \\
C_{O, o}^{(0)} \sim-\frac{I_{s s}(\beta)}{2 \pi \hat{\rho}} & & \hat{\rho} \rightarrow 0,
\end{array}
$$

and

$$
\begin{aligned}
\frac{\mathcal{D}}{\hat{\rho}} \frac{\partial^{2}}{\partial \hat{\rho}^{2}}\left(\hat{\rho} C_{R, o}^{(0)}\right) & =\frac{\partial C_{R, o}^{(0)}}{\partial T} & & z>0, \\
C_{R, o}^{(0)} & =0 & & T=0, \\
C_{R, o}^{(0)} & \rightarrow 0 & & \hat{\rho} \rightarrow \infty, \\
C_{R, o}^{(0)} & \sim \frac{I_{s s}(\beta)}{2 \pi \mathcal{D} \hat{\rho}} & & \hat{\rho} \rightarrow 0,
\end{aligned}
$$

whose solutions are:

$$
\begin{aligned}
& C_{O, o}^{(0)}=-\frac{I_{s s}(\beta)}{2 \pi \hat{\rho}} \operatorname{erfc}\left(\frac{\hat{\rho}}{2 \sqrt{T}}\right) \\
& C_{R, o}^{(0)}=\frac{I_{s s}(\beta)}{2 \pi \mathcal{D} \hat{\rho}} \operatorname{erfc}\left(\frac{\hat{\rho}}{2 \sqrt{\mathcal{D} T}}\right) .
\end{aligned}
$$

\subsubsection{First-order inner solution}

Next we apply Van Dyke's matching rule [40] to determine the first-order influence of the outer solution upon the inner problem. Writing (39) and (40) in terms of the inner variable $\hat{\rho}=\epsilon \rho$, where $\rho=\sqrt{r^{2}+z^{2}}$, and taking the first two terms of the expansion as $\epsilon \rightarrow 0$, we obtain

$$
\begin{aligned}
& C_{O, o}=-\frac{I_{s s}(\beta)}{2 \pi \rho}+\epsilon \frac{I_{s s}(\beta)}{2 \pi^{\frac{3}{2}} \sqrt{T}}+O\left(\epsilon^{3}\right), \\
& C_{R, o}=\frac{I_{s s}(\beta)}{2 \pi \mathcal{D} \rho}-\epsilon \frac{I_{s s}(\beta)}{2 \pi^{\frac{3}{2}} \mathcal{D}^{\frac{3}{2}} \sqrt{T}}+O\left(\epsilon^{3}\right) .
\end{aligned}
$$

Hence, the first-order terms $C_{O, i}^{(1)}$ and $C_{R, i}^{(1)}$ of the inner perturbation series, (31a) and (31b), satisfy:

$$
\nabla^{2} C_{O, i}^{(1)}=0, \quad \nabla^{2} C_{R, i}^{(1)}=0,
$$

with boundary conditions as $\rho \rightarrow \infty$ :

$$
C_{O, i}^{(1)} \rightarrow \frac{I_{s s}(\beta)}{2 \pi^{\frac{3}{2}} \sqrt{T}}, \quad C_{R, i}^{(1)} \rightarrow-\frac{I_{s s}(\beta)}{2 \pi^{\frac{3}{2}} \mathcal{D}^{\frac{3}{2}} \sqrt{T}} .
$$


On the electrode surface, $r \leq 1, z=0$, the boundary conditions are:

$$
\begin{aligned}
\frac{\partial C_{O, i}^{(1)}}{\partial z} & =k_{f} C_{O, i}^{(1)}-k_{b} C_{R, i}^{(1)}, \\
\frac{\partial C_{O, i}^{(1)}}{\partial z} & =-\mathcal{D} \frac{\partial C_{R, i}^{(1)}}{\partial z}
\end{aligned}
$$

For $r>1, z=0$, the no-flux condition is:

$$
\frac{\partial C_{O, i}^{(1)}}{\partial z}=\frac{\partial C_{R, i}^{(1)}}{\partial z}=0
$$

Using the governing equations, (43a), and the boundary conditions, (43b), (43d)-(43e), we see that the following quantity must be conserved:

$$
C_{O, i}^{(1)}+\mathcal{D} C_{R, i}^{(1)} \equiv-\frac{I_{s s}(\beta)}{2 \pi^{\frac{3}{2}} \sqrt{T}}\left(\mathcal{D}^{-\frac{1}{2}}-1\right)
$$

Hence $C_{R, i}^{(1)}$ can be eliminated from (43a)-(43e) to obtain a single problem for $C_{O, i}^{(1)}$ :

$$
\begin{array}{rlr}
\nabla^{2} C_{O, i}^{(1)} & =0 & z>0, \\
C_{O, i}^{(1)} \rightarrow \frac{I_{s s}(\beta)}{2 \pi^{\frac{3}{2}} \sqrt{T}} & \rho \rightarrow \infty, \\
-\frac{\partial C_{O, i}^{(1)}}{\partial z} & =\left\{\begin{array}{lll}
0 & r>1 & \\
\frac{k_{b} I_{s s}(\beta)}{2 \pi^{\frac{3}{2}} \mathcal{D} \sqrt{T}}\left(1-\mathcal{D}^{-\frac{1}{2}}\right)-\beta C_{O, i}^{(1)} & r \leq 1 & z=0 .
\end{array}\right.
\end{array}
$$

By comparison with (19), the solution to this problem can be written in terms of the steady-state solution, $C_{O}^{s s}$, as follows:

$$
C_{O, i}^{(1)}=\frac{I_{s s}(\beta)}{2 \pi^{\frac{3}{2}} \sqrt{T}}\left[1-\left(\frac{k_{f}+k_{b} \mathcal{D}^{-\frac{3}{2}}}{\beta}\right) C_{O}^{s s}(\beta ; r, z)\right] .
$$

From (44), we see that $C_{R, i}^{(1)}$ has the corresponding solution:

$$
C_{R, i}^{(1)}=-\frac{I_{s s}(\beta)}{2 \pi^{\frac{3}{2}} \mathcal{D} \sqrt{T}}\left[\mathcal{D}^{-\frac{1}{2}}-\left(\frac{k_{f}+k_{b} \mathcal{D}^{-\frac{3}{2}}}{\beta}\right) C_{O}^{s s}(\beta ; r, z)\right] .
$$

\subsection{Analytical expression for the long-time transient current}

Collecting the terms in the inner perturbation series for $C_{O, i}$, (33) and (46), the solution for $C_{O, i}$ is therefore given by

$$
\begin{array}{r}
C_{O, i}=C_{O}^{s s}(\beta ; r, z)+\frac{\epsilon}{\sqrt{T}} \frac{I_{s s}(\beta)}{2 \pi^{\frac{3}{2}}}\left[1-\frac{k_{f}+k_{b} \mathcal{D}^{-\frac{3}{2}}}{\beta} C_{O}^{s s}(\beta ; r, z)\right] \\
+O\left(\left(\frac{\epsilon}{\sqrt{T}}\right)^{3}\right)
\end{array}
$$


while the corresponding solution for $C_{R, i}$ is found from (34) and (47) to be:

$$
\begin{aligned}
& C_{R, i}=-\frac{1}{\mathcal{D}} C_{O}^{s s}(\beta ; r, z)-\frac{\epsilon}{\sqrt{T}} \frac{I_{s s}(\beta)}{2 \pi^{\frac{3}{2}} \mathcal{D}}\left[\mathcal{D}^{-\frac{1}{2}}-\left(\frac{k_{f}+k_{b} \mathcal{D}^{-\frac{3}{2}}}{\beta}\right) C_{O}^{s s}(\beta ; r, z)\right] \\
& +O\left(\left(\frac{\epsilon}{\sqrt{T}}\right)^{3}\right) \text {. }
\end{aligned}
$$

In expressions (48) and (49), we have indicated the error term of $O\left((\epsilon / \sqrt{T})^{3}\right)$. In other words, it transpires that the second-order corrections of $O\left(\epsilon^{2} / T\right)$ are identically zero. We relegate the detailed justification of this to Appendix B.

Expression (48) implies that the long-time transient current is given by

$$
\begin{aligned}
I(t) & =\left.2 \pi \int_{0}^{1} \frac{\partial C_{O, i}}{\partial z}\right|_{z=0} r \mathrm{~d} r \\
& =I_{s s}(\beta)\left[1-\frac{I_{s s}(\beta)}{2 \pi^{\frac{3}{2}} \sqrt{t}}\left(\frac{k_{f}+k_{b} \mathcal{D}^{-\frac{3}{2}}}{\beta}\right)\right]+O\left(t^{-\frac{3}{2}}\right),
\end{aligned}
$$

where $I_{s s}(\beta)$ is the steady-state current defined by (21) and we return to the physical time variable $t=T / \epsilon^{2}$. Converting back to dimensional variables gives the main result of this article:

$$
\begin{aligned}
& \tilde{I}(\tilde{t}) \sim n F \tilde{a} \tilde{D}_{O} \tilde{D}_{R} I_{s s}(\beta)\left(\frac{\tilde{k}_{f} \tilde{C}_{O}^{*}-\tilde{k}_{b} \tilde{C}_{R}^{*}}{\tilde{k}_{f} \tilde{D}_{R}+\tilde{k}_{b} \tilde{D}_{O}}\right) \times \\
& {\left[1-\frac{\tilde{a} I_{s s}(\beta)}{2 \pi^{\frac{3}{2}} \sqrt{\tilde{t} \tilde{D}_{O} \tilde{D}_{R}}}\left(\frac{\tilde{k}_{f} \tilde{D}_{R}^{\frac{3}{2}}+\tilde{k}_{b} \tilde{D}_{O}^{\frac{3}{2}}}{\tilde{k}_{f} \tilde{D}_{R}+\tilde{k}_{b} \tilde{D}_{O}}\right)\right], \quad \text { as } \tilde{t} \rightarrow \infty, }
\end{aligned}
$$

where the error in the formula is proportional to $\tilde{t}^{-3 / 2}$ and $\beta$ is defined as in (2).

\section{Results and discussion}

In this section, we consider special cases of the solution for the current response (52) and show that it encapsulates existing solutions in the literature for diffusionlimited currents. We also verify the analytical solution by comparison with numerically calculated currents.

\subsection{Special cases of the current response}

\subsubsection{Extreme polarisation currents}

For a reduction reaction, extreme polarisation corresponds to letting $k_{f} \rightarrow \infty$ and $k_{b} \rightarrow 0$. Since $I_{s s}(\infty)=-4$, the resulting time-dependent limiting current is given by:

$$
\tilde{I}(\tilde{t}) \sim-4 n F \tilde{a} \tilde{D}_{O} \tilde{C}_{O}^{*}\left[1+\frac{2 \tilde{a}}{\pi^{\frac{3}{2}} \sqrt{\tilde{t} \tilde{D}_{O}}}\right] .
$$


This result agrees with the first two terms of the series reported by Shoup and Szabo [19] and Phillips [20]. Similarly for an oxidation reaction, $k_{b} \rightarrow \infty$ and $k_{f} \rightarrow 0$, so that the limiting current is given by:

$$
\tilde{I}(\tilde{t}) \sim 4 n F \tilde{a} \tilde{D}_{R} \tilde{C}_{R}^{*}\left[1+\frac{2 \tilde{a}}{\pi^{\frac{3}{2}} \sqrt{\tilde{t} \tilde{D}_{R}}}\right] .
$$

\subsubsection{Reversible reactions}

Aoki and Osteryoung [17, 18] (corrected by Shoup and Szabo [19]) found the complete expansion using the Wiener-Hopf method for the special case when $\tilde{C}_{R}^{*}=0$, $\tilde{D}_{O}=\tilde{D}_{R}=\tilde{D}$ and $k_{f}, k_{b} \rightarrow \infty$ such that $k_{b} / k_{f}=O(1)$. Rajendran and Sangaranarayanan [23] also reported four terms of the series for the current. In this case, $I_{s s}(\beta)=-4$, and, making the same assumptions in (52), we obtain the same result as the first two terms in their series, namely

$$
\tilde{I}(\tilde{t}) \sim-4 n F \tilde{a} \tilde{D} \tilde{C}_{O}^{*}\left(1+\frac{\tilde{k}_{b}}{\tilde{k}_{f}}\right)^{-1}\left[1+\frac{2 \tilde{a}}{\pi^{\frac{3}{2}} \sqrt{\tilde{D} \tilde{t}}}\right] .
$$

If the diffusion coefficients are not the same and $\tilde{C}_{R}^{*} \neq 0$, then the generalised result for reversible reactions is given by

$$
\begin{aligned}
\tilde{I}(\tilde{t}) \sim-4 n F \tilde{a} \tilde{D}_{O} \tilde{D}_{R}\left(\frac{\tilde{k}_{f} \tilde{C}_{O}^{*}-\tilde{k}_{b} \tilde{C}_{R}^{*}}{\tilde{k}_{f} \tilde{D}_{R}+\tilde{k}_{b} \tilde{D}_{O}}\right) \times & \\
& {\left[1+\frac{2 \tilde{a}}{\pi^{\frac{3}{2}} \sqrt{\tilde{t} \tilde{D}_{O} \tilde{D}_{R}}}\left(\frac{\tilde{k}_{f} \tilde{D}_{R}^{\frac{3}{2}}+\tilde{k}_{b} \tilde{D}_{O}^{\frac{3}{2}}}{\tilde{k}_{f} \tilde{D}_{R}+\tilde{k}_{b} \tilde{D}_{O}}\right)\right] . }
\end{aligned}
$$

\subsubsection{Irreversible reactions}

For an irreversible reduction reaction, $k_{b} \rightarrow 0$, whilst $k_{f}$ remains $O(1)$, so that

$$
\tilde{I}(\tilde{t}) \sim n F \tilde{a} \tilde{D}_{O} I_{s s}\left(\frac{\tilde{k}_{f} \tilde{a}}{\tilde{D}_{O}}\right) \tilde{C}_{O}^{*}\left[1-\frac{\tilde{a} I_{s s}\left(\frac{\tilde{k}_{f} \tilde{a}}{\tilde{D}_{O}}\right)}{2 \pi^{\frac{3}{2}} \sqrt{\tilde{t} \tilde{D}_{O}}}\right] .
$$

For an irreversible oxidation reaction, $k_{f} \rightarrow 0$, whilst $k_{b}$ remains $O(1)$, so that

$$
\tilde{I}(\tilde{t}) \sim-n F \tilde{a} \tilde{D}_{R} I_{s s}\left(\frac{\tilde{k}_{b} \tilde{a}}{\tilde{D}_{R}}\right) \tilde{C}_{R}^{*}\left[1-\frac{\tilde{a} I_{s s}\left(\frac{\tilde{k}_{b} \tilde{a}}{\tilde{D}_{R}}\right)}{2 \pi^{\frac{3}{2}} \sqrt{\tilde{t} \tilde{D}_{R}}}\right] .
$$

\subsection{Comparison with numerical simulations}

To verify our prediction (51) for the long-time current response, we performed numerical simulations using the fully implicit finite-difference (FIFD) method detailed by Gavaghan [26, 27], with a spatial mesh expanding exponentially from the edge of the disk. The problem to be solved is given by the governing equations (12), with 
initial conditions (13), far-field boundary conditions (14) implemented at the edge of the finite domain, and boundary conditions on the electrode (15) and the surrounding insulator (16).

For the dimensionless problem, we chose the region of integration to be $(0 \leq r \leq$ $\left.101=r_{\max }, \quad 0 \leq z \leq 100=z_{\max }\right)$ and solved for $0 \leq t \leq 10=t_{\max }$. Note that the domain of integration was larger than the $6 \sqrt{D t_{\max }}$ condition recommended by Britz, [41], to ensure that the finite boundaries have no effect on the processes at the electrode; in this case, $r_{\max }, z_{\max }$ must be greater than $\max \left(6 \sqrt{t_{\max }}, 6 \sqrt{\mathcal{D} t_{\max }}\right)$. Following Gavaghan $[26,27]$, we chose the mesh parameters to be $h_{\text {last }}=8 \times 10^{-5}$ and $f=1.175$, and the time-stepping was chosen to ensure accurate solutions at all times. The initial time-step was taken to be $10^{-6}$ and was increased by a factor of 10 after every 1000 steps. To test whether this was sufficiently accurate, we also performed the simulations with an initial time-step of $10^{-5}$ and found that there was a negligible difference in the values of the current over the entire time domain; the maximum percentage difference was less than $0.5 \%$ for all the simulations run.

In Figure 3, we show comparisons of the numerical and analytical solutions for various combinations of the parameters $k_{f}, k_{b}$ and $\mathcal{D}$. We have plotted $I(t) / \beta$ against $t$ to ensure that the full effect of different diffusion coefficients on the current is captured, since the non-dimensionalisation of the concentrations includes a factor of $1 / \beta$, cf. expression (10b). The percentage differences between the numerical and analytical solutions are plotted in Figure 4, which confirms that the analytical solution diverges from the numerical solution for small $t$ and converges for large $t$. We expect that the analytical solution (51) is valid for long-times $t$ such that

$$
t \gg \max \left(1, \mathcal{D}^{-1}\right),
$$

which is the non-dimensional equivalent of condition (30). For the parameters considered in Figure 4, the percentage difference between the analytical and numerical solutions is less than $1.5 \%$ for $t \geq 1$.

\section{Conclusions}

We have derived a novel approximate solution (52) for the long-time-dependent chronoamperometric current at a circular disk electrode. The solution generalises previous results in the literature to allow for quasi-reversible reactions at the electrode. It also extends the previous work to allow the oxidant and the reductant to have different diffusion coefficients. We showed that our new solution encapsulates and generalises the known solutions for diffusion-limited currents, and agrees well with numerically calculated solutions. Our analysis shows that the large-time current decays to its steady-state value like $\tilde{t}^{-1 / 2}$ as $\tilde{t} \rightarrow \infty$. A key conclusion of our work is that the correction of $O\left(\tilde{t}^{-1}\right)$ is identically zero, so that a simple two-term approximation gives surprisingly accurate results.

We have made no assumptions in this article about the form of the forward and backward rate constants, $\tilde{k}_{f}$ and $\tilde{k}_{b}$, other than that they are constant. The most commonly used model for the forward and backward rate constants is the Butler-Volmer model [42], which relates the rate constants to the applied potential at the electrode 
surface. In the future, we plan to discuss how the results in this paper can be applied to define a protocol for estimating the parameters of the Butler-Volmer model from a series of chronoamperometric experiments, and we will verify the protocol experimentally.

\section{Acknowledgements}

Funding for this project was provided by the Engineering and Physical Sciences Research Council (EPSRC) (grant number EP/F044690/1) and is gratefully acknowledged.

\section{Supplementary Data}

We supply a working curve for the non-dimensional steady-state current $I_{s s}(\beta)$ as a function of $\beta$ for the range $0 \leq \beta \leq 500$, at the points $\beta_{j}=0.05 \times(j-1)$, $j=1, \ldots, 10,001$. Details of its calculation are described in Appendix A. The file is called 'Iss_working_curve.txt'.

\section{References}

[1] K. Aoki, Theory of ultramicroelectrodes, Electroanalysis 5 (1993) 627-639.

[2] C. Amatore, Electrochemistry at ultramicroelectrodes, in: I. Rubinstein (Ed.), Physical Electrochemistry: Principles, Methods and Applications, Marcel Dekker, New York, 1995, pp. 131-208.

[3] C.A. Basha, L. Rajendran, Theories of ultramicrodisc electrodes: review article, Int. J. Electrochem. Sci. 1 (2006) 268-282.

[4] A.M. Bond, K.B. Oldham, C.G. Zoski, Theory of electrochemical processes at an inlaid disc microelectrode under steady-state conditions, J. Electroanal. Chem. 245 (1988) 71-104.

[5] J. Newman, Resistance for flow of current to a disk, J. Electrochem. Soc. 113 (1966) 501-502.

[6] Y. Saito, A theoretical study on the diffusion current at the stationary electrodes of circular and narrow band types, Rev. Polarography 15 (1968) 177-187.

[7] I.N. Sneddon, Mixed Boundary Value Problems in Potential Theory, first ed., John Wiley \& Sons Inc., New York, 1966.

[8] K.B. Oldham, Steady-state concentrations and fluxes in the vicinity of a reversible inlaid disc microelectrode, J. Electroanal. Chem. 260 (1989) 461-467.

[9] C.G. Phillips, The steady-state current for a microelectrode near diffusion-limited conditions, J. Electroanal. Chem. 291 (1990) 251-256. 
[10] M.A. Bender, H.A. Stone, An integral equation approach to the study of the steady state current at surface microelectrodes, J. Electroanal. Chem. 351 (1993) 29-55.

[11] K. Aoki, K. Tokuda, H. Matsuda, Theory of stationary current-potential curves at microdisk electrodes for quasi-reversible and totally irreversible electrode reactions, J. Electroanal. Chem. 235 (1987) 87-96.

[12] M. Fleischmann, J. Daschbach, S. Pons, The behavior of microdisk and microring electrodes. Application of Neumann's integral theorem to the prediction of the steady state response of microdisks, J. Electroanal. Chem. 263 (1989) 189-203.

[13] J. Daschbach, S. Pons, M. Fleischmann, The behavior of microdisk and microring electrodes. Application of Neumann's integral theorem to the prediction of the steady state response of microdisks. Numerical illustrations, J. Electroanal. Chem. 263 (1989) 205-224.

[14] D.R. Baker, M.W. Verbrugge, An integral-transform formulation for the reaction distribution on a stationary-disk electrode below the limiting current, J. Electrochem. Soc. 137 (1990) 1832-1842.

[15] D.R. Baker, M.W. Verbrugge, An analytic solution for the microdisk electrode and its use in the evaluation of charge-transfer rate constants, J. Electrochem. Soc. 137 (1990) 3836-3845.

[16] K.B. Oldham, C.G. Zoski, Steady-state voltammetry at an inlaid microdisc: comparison of three approaches, J. Electroanal. Chem. 313 (1991) 17-28.

[17] K. Aoki, J. Osteryoung, Diffusion-controlled current at the stationary finite disk electrode. Theory, J. Electroanal. Chem. 122 (1981) 19-35.

[18] K. Aoki, J. Osteryoung, Formulation of the diffusion-controlled current at very small stationary disk electrodes, J. Electroanal. Chem. 160 (1984) 335-339.

[19] D. Shoup, A. Szabo, Chronoamperometric current at finite disk electrodes, J. Electroanal. Chem. 140 (1982) 237-245.

[20] C.G. Phillips, The long-time transient of two- and three-dimensional diffusion in microelectrode chronoamperometry, J. Electroanal. Chem. 333 (1992) 11-32.

[21] C.G. Phillips, K.M. Jansons, The short-time transient of diffusion outside a conducting body, Proc. Roy. Soc. A 428 (1990) 431-449.

[22] K.B. Oldham, Edge effects in semiinfinite diffusion, J. Electroanal. Chem. 122 (1981) 1-17.

[23] L. Rajendran, M.V. Sangaranarayanan, Diffusion at ultramicro disk electrodes: chronoamperometric current for steady-state $\mathrm{Ec}^{\prime}$ reaction using scattering analogue techniques, J. Phys. Chem. B 103 (1999) 1518-1524. 
[24] M. Fleischmann, J. Daschbach, S. Pons, The behavior of microdisk and microring electrodes. Mass transport to the disk in the unsteady state. Chronoamperometry, J. Electroanal. Chem. 250 (1988) 269-276.

[25] M. Fleischmann, D. Pletcher, G. Denault, J. Daschbach, S. Pons, The behavior of microdisk and microring electrodes. Prediction of the chronoamperometric response of microdisks and of the steady state for CE and EC catalytic reactions by application of Neumann's integral theorem, J. Electroanal. Chem. 263 (1989) 225-236.

[26] D.J. Gavaghan, An exponentially expanding mesh ideally suited to the fast and efficient simulation of diffusion processes at microdisc electrodes. 1. Derivation of the mesh, J. Electroanal. Chem. 456 (1998) 1-12.

[27] D.J. Gavaghan, An exponentially expanding mesh ideally suited to the fast and efficient simulation of diffusion processes at microdisc electrodes. 2. Application to chronoamperometry, J. Electroanal. Chem. 456 (1998) 13-23.

[28] K. Harriman, D.J. Gavaghan, P. Houston, E. Süli, Adaptative finite element simulation of currents at microelectrodes to a guaranteed accuracy. Application to a simple model problem, Electrochem. Comm. 2 (2000) 150-156.

[29] K. Harriman, D.J. Gavaghan, P. Houston, E. Süli, Adaptive finite element simulation of currents at microelectrodes to a guaranteed accuracy. Theory, Electrochem. Comm. 2 (2000) 157-162.

[30] K. Harriman, D.J. Gavaghan, E. Süli, Adaptive finite element simulation of chronoamperometry at microdisc electrodes, Electrochem. Comm. 5 (2003) 519529 .

[31] A. Oleinick, C. Amatore, I. Svir, Efficient quasi-conformal map for simulation of diffusion at disk microelectrodes, Electrochem. Comm. 6 (2004) 588-594.

[32] C. Amatore, A.I. Oleinick, I. Svir, Construction of optimal quasi-conformal mappings for the 2D-numerical simulation of diffusion at microelectrodes. Part 1: Principle of the method and its application to the inlaid disk microelectrode, J. Electroanal. Chem. 597 (2006) 69-76.

[33] C. Amatore, A.I. Oleinick, I. Svir, Construction of optimal quasi-conformal mappings for the 2D numerical simulation of diffusion at microelectrodes. Part 2. Application to recessed or protruding electrodes and their arrays, J. Electroanal. Chem. 597 (2006) 77-85.

[34] C. Amatore, A.I. Oleinick, I. Svir, Theoretical analysis of microscopic ohmic drop effects on steady-state and transient voltammetry at the disk microelectrode: a quasi-conformal mapping modeling and simulation, Anal. Chem. 80 (2008) 7947-7956. 
[35] M.V. Mirkin, A.J. Bard, Multidimensional integral equations. Part 1. A new approach to solving microelectrode diffusion problems, J. Electroanal. Chem. 323 (1992) 1-27.

[36] C.J. Tranter, On some dual integral equations occurring in potential problems with axial symmetry, Quart. J. Mech. and Appl. Math. 4 (1950) 411-419.

[37] K. Nisanciog̈lu, J. Newman, The short-time response of a disk electrode, J. Electrochem. Soc. 121 (1974) 523-527.

[38] J. Newman, The Fundamental Principles of Current Distribution and Mass Transport in Electrochemical Cells, in: A. J. Bard (Ed.), Electroanalytical Chemistry, Vol. 6, Marcel Dekker Inc., New York, 1973, pp. 187-352.

[39] M. Abramowitz, I.A. Stegun, (Eds.) Handbook of Mathematical Functions with Formulas, Graphs, and Mathematical Tables, National Bureau of Standards Applied Mathematics Series, 55, U.S. Government Printing Office, Washington, DC, 1964.

[40] E.J. Hinch, Perturbation Methods, first ed., Cambridge University Press, Cambridge, 1991.

[41] D. Britz, Digital Simulation in Electrochemistry, second ed., Springer, Heidelberg, 1988 .

[42] A.J. Bard, L.R. Faulkner, Electrochemical Methods: Fundamentals and Applications, second ed., John Wiley \& Sons, New York, 2001.

\section{Appendix A. Computing the steady-state non-dimensional current $I_{s s}(\beta)$}

To recap for clarity, the solution for the steady-state oxidant concentration, $C_{O}^{s s}(\beta ; r, z)$, satisfies the following problem (cf. (19)):

$$
\begin{array}{rlrl}
\nabla^{2} C_{O}^{s s} & =0 & & z>0, \\
C_{O}^{s s} & \rightarrow 0 & & z \rightarrow \infty, \\
\frac{\partial C_{O}^{s s}}{\partial z} & =q(\beta ; r)=\left\{\begin{array}{lll}
0 & r>1, & \\
\beta\left(C_{O}^{s s}-1\right) & r \leq 1, &
\end{array}\right.
\end{array}
$$

The non-dimensional steady-state current through the disk electrode is given by

$$
I_{s s}(\beta)=2 \pi \int_{0}^{1} \frac{\partial C_{O}^{s s}}{\partial z}(\beta ; r, 0) r \mathrm{~d} r=2 \pi \int_{0}^{1} q(\beta ; r) r \mathrm{~d} r .
$$

Let $\widehat{q}(\beta ; s)$ denote the Hankel transform of $q(\beta ; r)$, namely

$$
\widehat{q}(\beta ; s)=\int_{0}^{\infty} q(\beta ; r) J_{0}(r s) r \mathrm{~d} r .
$$


It is straightforward to deduce from (A.1) and (A.2) that $\widehat{q}(\beta ; s)$ must satisfy the dual integral equations

$$
\begin{cases}\int_{0}^{\infty} \widehat{q}(\beta ; s)(\beta+s) J_{0}(r s) \mathrm{d} s=-\beta & r \leq 1, \\ \int_{0}^{\infty} \widehat{q}(\beta ; s) J_{0}(r s) s \mathrm{~d} s=0 & r>1 .\end{cases}
$$

Once $\widehat{q}(\beta ; s)$ is determined, we can compute $I_{s s}(\beta)$ using

$$
I_{s s}(\beta)=2 \pi \widehat{q}(\beta ; 0) .
$$

We solve (A.4) to find $\widehat{q}(\beta ; s)$ using Tranter's method [36]. If we decompose $\widehat{q}(\beta ; s)$ into a series of the form

$$
\widehat{q}(\beta ; s)=\frac{1}{s} \sum_{n=0}^{\infty} a_{n} J_{2 n+1}(s),
$$

then (A.4b) is satisfied identically, while (A.4a) leads to an infinite system of linear algebraic equations for the coeffients $a_{n}$. We truncate the system at some large finite size $N$ and hence obtain a matrix equation of the form

$$
\sum_{n=0}^{N-1}\left(\delta_{m n}+\beta L_{m n}\right) a_{n}=-\beta \delta_{m 0}
$$

for $a_{0}, a_{1}, \cdots, a_{N-1}$, where

$$
L_{m n}=\frac{8(-1)^{m+n}(2 m+1)}{\pi(2 m+2 n+1)(2 m-2 n+1)(2 n-2 m+1)(2 m+2 n+3)},
$$

and $\delta_{m n}$ is the Kronecker delta. For each finite value of $\beta$ and $N$, (A.7) is easily inverted and the non-dimensional steady-state current is then recovered from

$$
I_{s s}^{(N)}(\beta)=\pi a_{0}
$$

Accurate computation of $I_{s s}(\beta)$ requires an estimate of the truncation error in $I_{s s}^{(N)}(\beta)$. Assuming that the error is proportional to $N^{-p}$ for some positive integer $p$, it is possible to determine that the relative error, $\operatorname{Err}_{N}(\beta)$, decreases as $N^{-6}$, where $\operatorname{Err}_{N}(\beta)$ is defined as:

$$
\operatorname{Err}_{N}(\beta)=\frac{\left|I_{s s}^{(N)}(\beta)-I_{s s}(\beta)\right|}{\left|I_{s s}(\beta)\right|}
$$

We display a $\log$ - $\log$ plot of $\operatorname{Err}_{N}(\beta)$ versus $N$ for $\beta=500$ in Figure A.1; the dashed line indicates the slope of -6 .

For practical purposes, it is useful to have a working curve for $I_{s s}(\beta)$. We have calculated a working curve for $I_{s s}(\beta)$ using $N=50$ for $0 \leq \beta \leq 500$ at the 
points $\beta=\beta_{j}$, where $\beta_{j}=0.05(j-1), j=1, \ldots, 10,001$. The curve is plotted in Figure 2 in the main text and is supplied as Supplementary Data in a file called

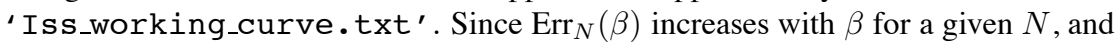
Figure A.1 shows that $\operatorname{Err}_{N}(500)=O\left(10^{-7}\right)$ at $N=50$, this implies that the relative error at each point on the calculated curve is less than $O\left(10^{-7}\right)$.

Finally, we note that the asymptotic approximation, (23), derived by Phillips [21] can be used to calculate $I_{s s}(\beta)$ for larger values of $\beta$; for $\beta>500$, the error is less than $0.2 \%$.

\section{Appendix B. Higher-order terms in the inner perturbation series solution (31)}

In the main body of the text we found the first two terms in the inner perturbation expansions for $C_{O, i}(r, z, T)$ and $C_{R, i}(r, z, T)$, (31). The leading-order solutions $C_{O, i}^{(0)}$ and $C_{R, i}^{(0)}$ are given by (33) and (34) respectively, while the first-order solutions $C_{O, i}^{(1)}$ and $C_{R, i}^{(1)}$ are given by (46) and (47). We have also found the leading-order terms in the outer perturbation expansions for $C_{O, o}(r, z, T)$ and $C_{R, o}(r, z, T),(32) ; C_{O, o}^{(0)}$ is given by (39) and $C_{R, o}^{(0)}$ is given by (40).

In this appendix, we continue the asymptotic matching procedure to find the firstorder outer solutions, $C_{O, o}^{(1)}$ and $C_{R, o}^{(1)}$. Subsequent matching back to the inner solution shows that the second-order inner solutions $C_{O, i}^{(2)}$ and $C_{R, i}^{(2)}$ are zero. This implies that the error in the inner perturbation expansions truncated at two terms is $O\left((\epsilon / \sqrt{T})^{3}\right)$ (or equivalently $O\left(t^{-3 / 2}\right)$ ), as we have indicated in expressions (48), (49) and (51).

\section{Appendix B.1. First-order outer solution}

Using the method of Van Dyke and the far-field behaviour of $C_{O}^{s s}(\beta ; r, z)$ given by (27), we find that the two-term outer expansion of the two-term inner solution is given by:

$$
\begin{aligned}
C_{O, i}=-\epsilon \frac{I_{s s}(\beta)}{2 \pi}\left(\frac{1}{\hat{\rho}}-\frac{1}{\sqrt{\pi T}}\right)+\epsilon^{2}\left(k_{f}+k_{b} \mathcal{D}^{-\frac{3}{2}}\right) \frac{I_{s s}(\beta)^{2}}{4 \pi^{\frac{5}{2}} \beta \sqrt{T} \hat{\rho}}+O\left(\epsilon^{3}\right) \\
C_{R, i}=\epsilon \frac{I_{s s}(\beta)}{2 \pi \mathcal{D}}\left(\frac{1}{\hat{\rho}}-\left(\frac{1}{\mathcal{D} \pi T}\right)^{\frac{1}{2}}\right) \\
\quad-\epsilon^{2}\left(k_{f}+k_{b} \mathcal{D}^{-\frac{3}{2}}\right) \frac{I_{s s}(\beta)^{2}}{4 \pi^{\frac{5}{2}} \mathcal{D} \beta \sqrt{T} \hat{\rho}}+O\left(\epsilon^{3}\right) .
\end{aligned}
$$


Hence the outer solutions, $C_{O, o}^{(1)}$ and $C_{R, o}^{(1)}$, must satisfy the following problems:

$$
\begin{array}{rlrl}
\frac{1}{\hat{\rho}} \frac{\partial^{2}}{\partial \hat{\rho}^{2}}\left(\hat{\rho} C_{O, o}^{(1)}\right) & =\frac{\partial C_{O, o}^{(1)}}{\partial T} & & z>0, \\
C_{O, o}^{(1)} & =0 & & T=0, \\
C_{O, o}^{(1)} & \rightarrow 0 & \hat{\rho} \rightarrow \infty, \\
C_{O, o}^{(1)} & \sim\left(k_{f}+k_{b} \mathcal{D}^{-\frac{3}{2}}\right) \frac{I_{s s}(\beta)^{2}}{4 \pi^{\frac{5}{2}} \beta \sqrt{T} \hat{\rho}} & & \hat{\rho} \rightarrow 0,
\end{array}
$$

and

$$
\begin{array}{rlrl}
\frac{\mathcal{D}}{\hat{\rho}} \frac{\partial^{2}}{\partial \hat{\rho}^{2}}\left(\hat{\rho} C_{R, o}^{(1)}\right) & =\frac{\partial C_{R, o}^{(1)}}{\partial T} & & z>0, \\
C_{R, o}^{(1)} & =0 & & T=0, \\
C_{R, o}^{(1)} & \rightarrow 0 & \hat{\rho} \rightarrow \infty, \\
C_{R, o}^{(1)} & \sim-\left(k_{f}+k_{b} \mathcal{D}^{-\frac{3}{2}}\right) \frac{I_{s s}(\beta)^{2}}{4 \pi^{\frac{5}{2}} \mathcal{D} \beta \sqrt{T} \hat{\rho}} & \hat{\rho} & \rightarrow 0,
\end{array}
$$

and the solutions are found to be:

$$
\begin{aligned}
& C_{O, o}^{(1)}=\left(k_{f}+k_{b} \mathcal{D}^{-\frac{3}{2}}\right) \frac{I_{s s}(\beta)^{2}}{4 \pi^{\frac{5}{2}} \beta \sqrt{T} \hat{\rho}} e^{-\frac{\hat{\rho}^{2}}{4 T}}, \\
& C_{R, o}^{(1)}=-\left(k_{f}+k_{b} \mathcal{D}^{-\frac{3}{2}}\right) \frac{I_{s s}(\beta)^{2}}{4 \pi^{\frac{5}{2}} \mathcal{D} \beta \sqrt{T} \hat{\rho}} e^{-\frac{\hat{\rho}^{2}}{4 \mathcal{D} T}} .
\end{aligned}
$$

\section{Appendix B.2. Second-order inner solution}

The three-term inner expansion of the two-term outer solution is given by:

$$
\begin{aligned}
C_{O, o}=-\frac{I_{s s}(\beta)}{2 \pi \rho} & +\epsilon \frac{I_{s s}(\beta)}{2 \pi^{\frac{3}{2}} \sqrt{T}}\left(1+\left(k_{f}+k_{b} \mathcal{D}^{-\frac{3}{2}}\right) \frac{I_{s s}(\beta)}{2 \pi \beta \rho}\right) \\
& -\epsilon^{3} \frac{I_{s s}(\beta)}{2(\pi T)^{\frac{3}{2}}}\left[\frac{\rho^{2}}{12}+\left(k_{f}+k_{b} \mathcal{D}^{-\frac{3}{2}}\right) \frac{I_{s s}(\beta) \rho}{8 \pi \beta}\right]+O\left(\epsilon^{5}\right),
\end{aligned}
$$

and

$$
\begin{aligned}
C_{R, o}= & \frac{I_{s s}(\beta)}{2 \pi \mathcal{D} \rho}-\epsilon \frac{I_{s s}(\beta)}{2 \pi^{\frac{3}{2}} \mathcal{D} \sqrt{T}}\left(\mathcal{D}^{-\frac{1}{2}}+\left(k_{f}+k_{b} \mathcal{D}^{-\frac{3}{2}}\right) \frac{I_{s s}(\beta)}{2 \pi \beta \rho}\right) \\
& +\epsilon^{3} \frac{I_{s s}(\beta)}{2 \mathcal{D}^{2}(\pi T)^{\frac{3}{2}}}\left[\mathcal{D}^{-\frac{1}{2}} \frac{\rho^{2}}{12}+\left(k_{f}+k_{b} \mathcal{D}^{-\frac{3}{2}}\right) \frac{I_{s s}(\beta) \rho}{8 \pi \beta}\right]+O\left(\epsilon^{5}\right) .
\end{aligned}
$$

Since the coefficient of $\epsilon^{2}$ is zero, this means that $C_{O, i}^{(2)}$ and $C_{R, i}^{(2)}$ must satisfy linear homogeneous boundary-value problems, whose solutions are $C_{O, i}^{(2)}=C_{R, i}^{(2)}=0$, and hence there is no term of $O\left(\epsilon^{2}\right)$ in the inner solution. This allows us to deduce that the error in the inner perturbation expansion truncated at two terms is $O\left((\epsilon / \sqrt{T})^{3}\right)$ (or equivalently $O\left(t^{-3 / 2}\right)$ ). 


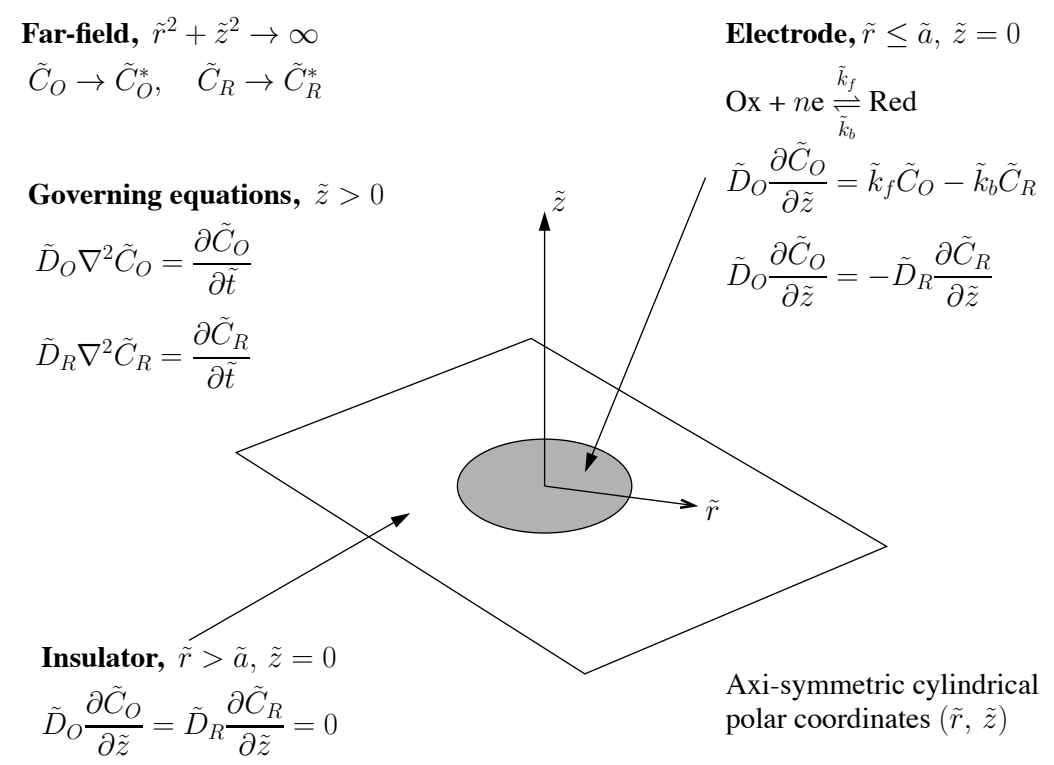

Figure 1: Schematic of the theoretical problem of an oxidant and reductant diffusing above a circular disk electrode of radius $\tilde{a}(\mathrm{~m})$ inlaid into an otherwise insulating plane at $\tilde{z}=0$. The concentration fields of the two species are denoted by $\tilde{C}_{O}(\tilde{r}, \tilde{z}, \tilde{t})$ and $\tilde{C}_{R}(\tilde{r}, \tilde{z}, \tilde{t})\left(\mathrm{mol} \mathrm{m}^{-3}\right)$ respectively, and their bulk concentrations in the far-field, $\tilde{C}_{O}^{*}$ and $\tilde{C}_{R}^{*}\left(\mathrm{~mol} \mathrm{~m}^{-3}\right)$, are constant. Their diffusion coefficients are represented by $\tilde{D}_{O}$ and $\tilde{D}_{R}\left(\mathrm{~m}^{2} \mathrm{~s}^{-1}\right)$. A redox reaction with forward and backward reaction rates denoted by $\tilde{k}_{f}$ and $\tilde{k}_{b}$ $\left(\mathrm{m} s^{-1}\right)$ takes place at the electrode, where the two species exchange $n$ electrons.

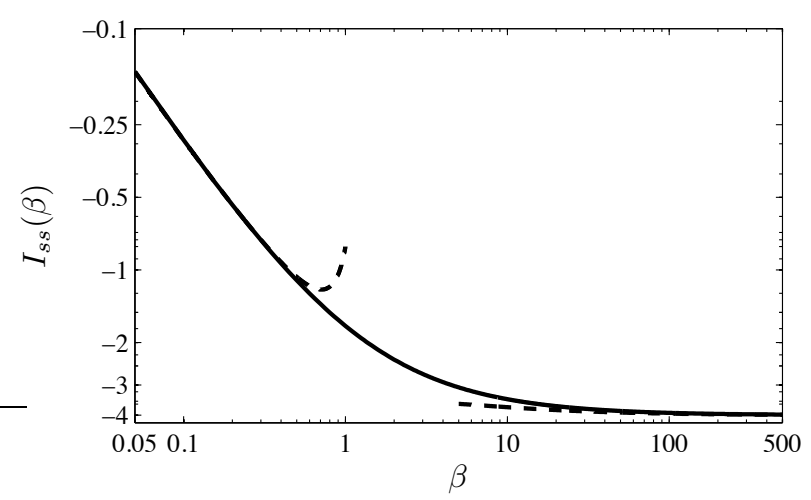

Figure 2: Log-log plot of the steady-state non-dimensional current, $I_{s s}(\beta)$, through a circular disk versus the mass transfer coefficient $\beta \in[0.05,500]$ (solid lines). The asymptotic approximations given by (22) as $\beta \rightarrow 0$ and (23) as $\beta \rightarrow \infty$ are shown as dashed curves. 


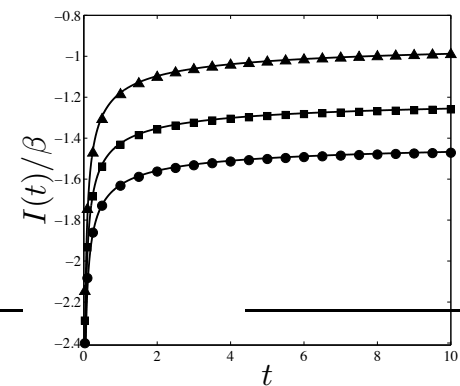

(a) Parameters $k_{f}=1, k_{b}=1$

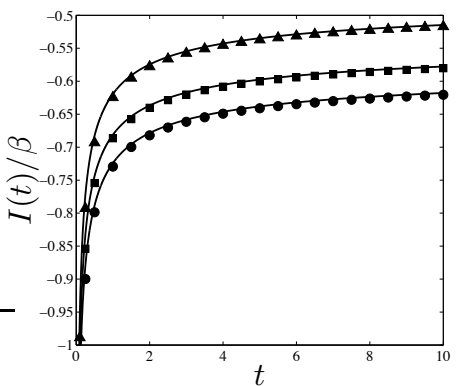

(b) Parameters $k_{f}=5, k_{b}=1$

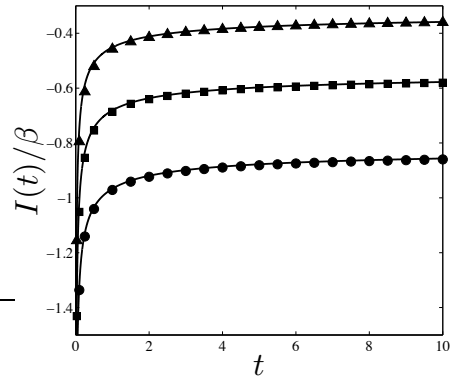

(c) Parameters $k_{f}=1, k_{b}=5$

Figure 3: Comparison between the non-dimensional analytical solution (solid lines) for the long-time transient current $I(t) / \beta$, where $I(t)$ is given by $(51)$, with numerically simulated values (triangles, squares and circles) using the FIFD method devised by Gavaghan [26, 27]. The three separate transients marked by the triangles, squares and circles on each plot correspond to taking $\mathcal{D}=0.5,1$, and 2 respectively and illustrate the impact of unequal diffusion coefficients. 


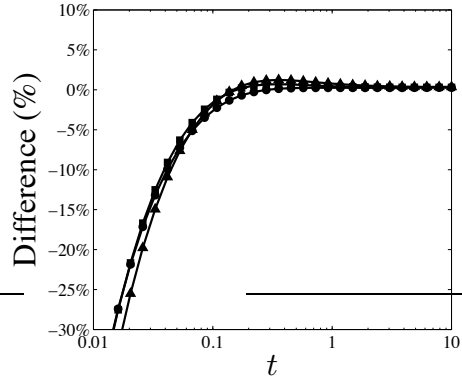

(a) Parameters $k_{f}=1, k_{b}=1$

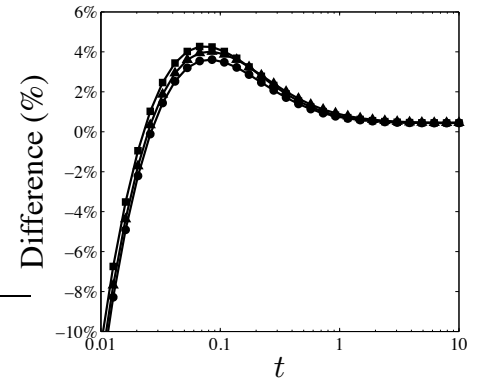

(b) Parameters $k_{f}=5, k_{b}=1$

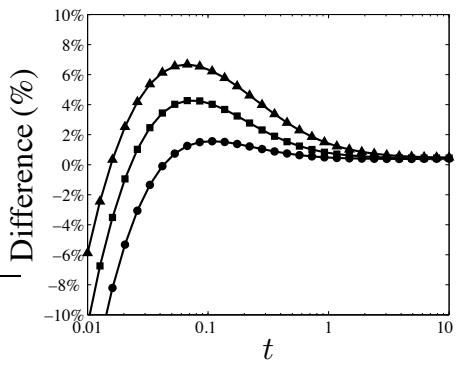

(c) Parameters $k_{f}=1, k_{b}=5$

Figure 4: Semi-log plots of the time-varying percentage difference between the non-dimensional long-time analytical solution for $I(t)$ given by (51) and numerically simulated values. The triangles, squares and circles denote different ratios of the diffusion coefficients, $\mathcal{D}=0.5,1,2$ respectively. As expected, the analytical solution diverges from the numerical solution at small times and converges at large times. For all the parameters considered, the percentage difference between the solutions is less than $1.5 \%$ for $t \geq 1$.

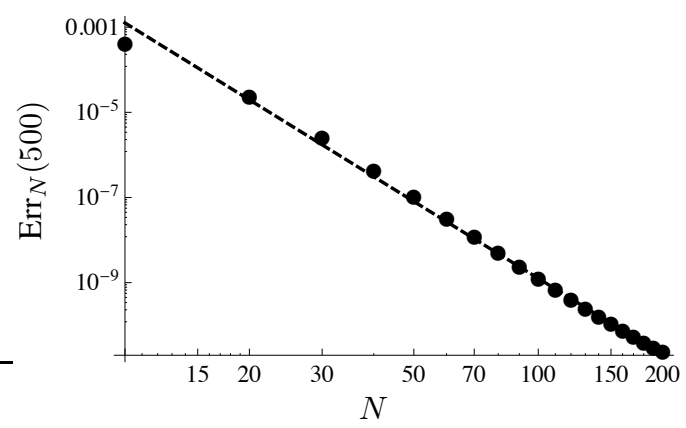

Figure A.1: $\log -\log$ plot of the relative error, $\operatorname{Err}_{N}(500)$, defined in (A.10), versus size $N$ of the truncated matrix equation (A.7). The dashed line indicates that the error decreases as $N^{-6}$. We note that choosing $N=50$ will ensure that the relative error in $I_{s s}^{(N)}(500)$ is $O\left(10^{-7}\right)$. 